medRxiv preprint doi: https://doi.org/10.1101/2020.08.18.20177329; this version posted September 10, 2020. The copyright holder for this preprint (which was not certified by peer review) is the author/funder, who has granted medRxiv a license to display the preprint in

It is made available under a CC-BY-NC-ND 4.0 International license .

\title{
A co-infection model for Two-Strain Malaria and Cholera with Optimal Control
}

\author{
K. U. Egeonu ${ }^{a}$, S. C. Inyama ${ }^{b}$ and A. Omame ${ }^{c, \dagger}$
}

प

\begin{abstract}
A mathematical model for two strains of Malaria and Cholera with optimal control is studied and analyzed to assess the impact of treatment controls in reducing the burden of the diseases in a population, in the presence of malaria drug resistance. The model is shown to exhibit the dynamical property of backward bifurcation when the associated reproduction number is less than unity. The global asymptotic stability of the disease-free equilibrium of the model is proven not to exist. The necessary conditions for the existence of optimal control and the optimality system for the model is established using the Pontryagin's Maximum Principle. Numerical simulations of the optimal control model reveal that malaria drug resistance can greatly influence the co-infection cases averted, even in the presence of treatment controls for co-infected individuals.
\end{abstract}

Keywords: Drug-resistance, Malaria, Cholera, Stability, Optimal control.

\footnotetext{
${ }^{\dagger}$ Corresponding author: omame2020@gmail.com, andrew.omame@futo.edu.ng
} 
medRxiv preprint doi: https://doi.org/10.1101/2020.08.18.20177329; this version posted September 10, 2020. The copyright holder for this preprint (which was not certified by peer review) is the author/funder, who has granted medRxiv a license to display the preprint in

It is made available under a CC-BY-NC-ND 4.0 International license .

\section{Introduction}

Malaria is one of the febrile illnesses and the most common deadly disease in the world caused by one or more species of plasmodium. These are Plasmodium falciparum, Plasmodium vivax, Plasmodium ovale, Plasmodium malariae, and Plasmodium knowlesi. Approximately half of the world population is at risk of malaria. Most of malaria cases and deaths occur in sub-Saharan Africa [1. According to the World Malaria Report 2018, there were about 228 million cases of malaria and an estimated 405000 deaths in 2018 [2]. More than 50\% of all cases were reported in six countries in Africa, with Nigeria recording $25 \%$ of the total global cases. Other African countries include Democratic Republic of Congo (12\%); Uganda (5\%); Cote d'Ivoire, Mozambique and Niger (4\% each) [2].

Cholera is an acute, diarrheal disease caused by infection of the intestine with the toxigenic bacterium Vibrio cholerae serogroup 01 or O139. About 2.9 million persons are estimated to be infected worldwide, with roughly 95,000 deaths annually [3. Mostly, the infection is mild or without symptoms, although can sometimes be severe. "Approximately $10 \%$ of those infected with the cholera will develop severe disease characterized by profuse watery diarrhea, vomiting, and leg cramps. In these people, rapid loss of body fluids leads to dehydration and shock. Without treatment, death can occur within hours" [3].

Mathematical models have been used extensively in studying the behaviour of infectious diseases [4, 5, 6, 7, 8, 9, 10, 11. A lot of models have been developed for the dynamics of the co-infections of two diseases [12, 13, 14, 15, 16, 17, 18. In this study, we shall be investigating the impact of malaria drug resistance on the co-infection malaria and cholera, using a mathematical model. Furthermore, optimal control analysis shall be carried out on the model to assess the impact of some control strategies.

\section{Model formulation}

The total human population at time $t$, denoted by $N_{\mathrm{H}}(t)$, is divided into eleven mutually exclusive classes, namely: susceptible humans $\left(S_{\mathrm{H}}(t)\right)$, untreated individuals with the sensitive malaria strain $\left(I_{\mathrm{US}}^{\mathrm{M}}(t)\right)$, individuals treated of sensitive malaria strain $\left(I_{\mathrm{TS}}^{\mathrm{M}}(t)\right)$, infectious individuals with resistant malaria strain $\left(I_{\mathrm{R}}^{\mathrm{M}}(t)\right)$, individual that recovered from malaria infection $\left.R_{\mathrm{M}}(t)\right)$, indivdiduals with cholera infection $\left(I_{\mathrm{C}}(t)\right)$, individuals who have recovered from cholera $\left(R_{\mathrm{C}}(t)\right)$, individuals untreated of sensitive malaria and infected with cholera, $\left(I_{\mathrm{USC}}^{\mathrm{M}}(t)\right)$, infectious individual treated of sensitive malaria but with cholera infection $\left(I_{\mathrm{TSC}}^{\mathrm{M}}(t)\right)$, individual with drug resistant malaria and cholera $\left(I_{\mathrm{RC}}^{\mathrm{M}}(t)\right)$, individuals who have recovered from both malaria and cholera $\left(R_{\mathrm{MC}}(t)\right)$. Thus

$$
N_{\mathrm{H}}(t)=S_{\mathrm{H}}(t)+I_{\mathrm{US}}^{\mathrm{M}}(t)+I_{\mathrm{TS}}^{\mathrm{M}}(t)+I_{\mathrm{R}}^{\mathrm{M}}(t)+R_{\mathrm{M}}(t)+I_{\mathrm{C}}(t)+R_{\mathrm{C}}(t)+I_{\mathrm{USC}}^{\mathrm{M}}(t)+I_{\mathrm{TSC}}^{\mathrm{M}}(t)+I_{\mathrm{RC}}^{\mathrm{M}}(t)+R_{\mathrm{MC}}(t)
$$

The total vector population $N_{\mathrm{v}}$ is divided int three mutually exclusive classes: susceptible vectors, $S_{\mathrm{v}}$, vectors infected with the sensitive malaria strain, $I_{\mathrm{vs}}$, vectors infected with the resistant malaria strain $I_{\mathrm{VR}}$. Thus, $N_{\mathrm{v}}=S_{\mathrm{v}}+I_{\mathrm{VS}}+I_{\mathrm{VR}}$. Also, the bacteria population is given by $\left(B_{\mathrm{C}}\right)$. Hence, the two strain malaria-cholera co-infection model is given by the following system of deterministic differential equations (the flow diagram of the model is shown in Figure 1 and the associated variables and parameters of the model are presented in Tables 1 and 2 , respectively): 
medRxiv preprint doi: https://doi.org/10.1101/2020.08.18.20177329; this version posted September 10, 2020. The copyright holder for this preprint (which was not certified by peer review) is the author/funder, who has granted medRxiv a license to display the preprint in

perpetuity.
It is made available under a CC-BY-NC-ND 4.0 International license .

Table 1: Description of variables in the model equation

\begin{tabular}{l|l}
\hline Variable & Interpretation \\
\hline$S_{\mathrm{H}}$ & Susceptible humans \\
$N_{\mathrm{H}}$ & Human population \\
$I_{\mathrm{US}}^{\mathrm{M}}$ & Individuals with sensitive malaria strain \\
$I_{\mathrm{TS}}^{\mathrm{M}}$ & Individuals treated for malaria infection \\
$R_{\mathrm{M}}$ & Individuals who have recovered from malaria infection \\
$I_{\mathrm{C}}$ & Individuals with cholera infection \\
$R_{\mathrm{C}}$ & Individuals who have recovered from cholera \\
$I_{\mathrm{USC}}^{\mathrm{M}}$ & Individuals dually infected with sensitive malaria and Cholera \\
$I_{\mathrm{TSC}}^{\mathrm{M}}$ & Individuals who are treated of sensitive malaria and infected with cholera \\
$I_{\mathrm{RC}}^{\mathrm{M}}$ & Individuals co-infected with resistant malaria and Cholera \\
$R_{\mathrm{MC}}$ & Individuals who have recovered from both malaria and cholera infections \\
$S_{\mathrm{V}}$ & Susceptible vectors \\
$I_{\mathrm{VR}}$ & Vectors with resistant malaria strain \\
$I_{\mathrm{VS}}$ & Vectors with sensitive malaria strain \\
$B_{\mathrm{C}}$ & Bacteria population \\
\hline
\end{tabular}

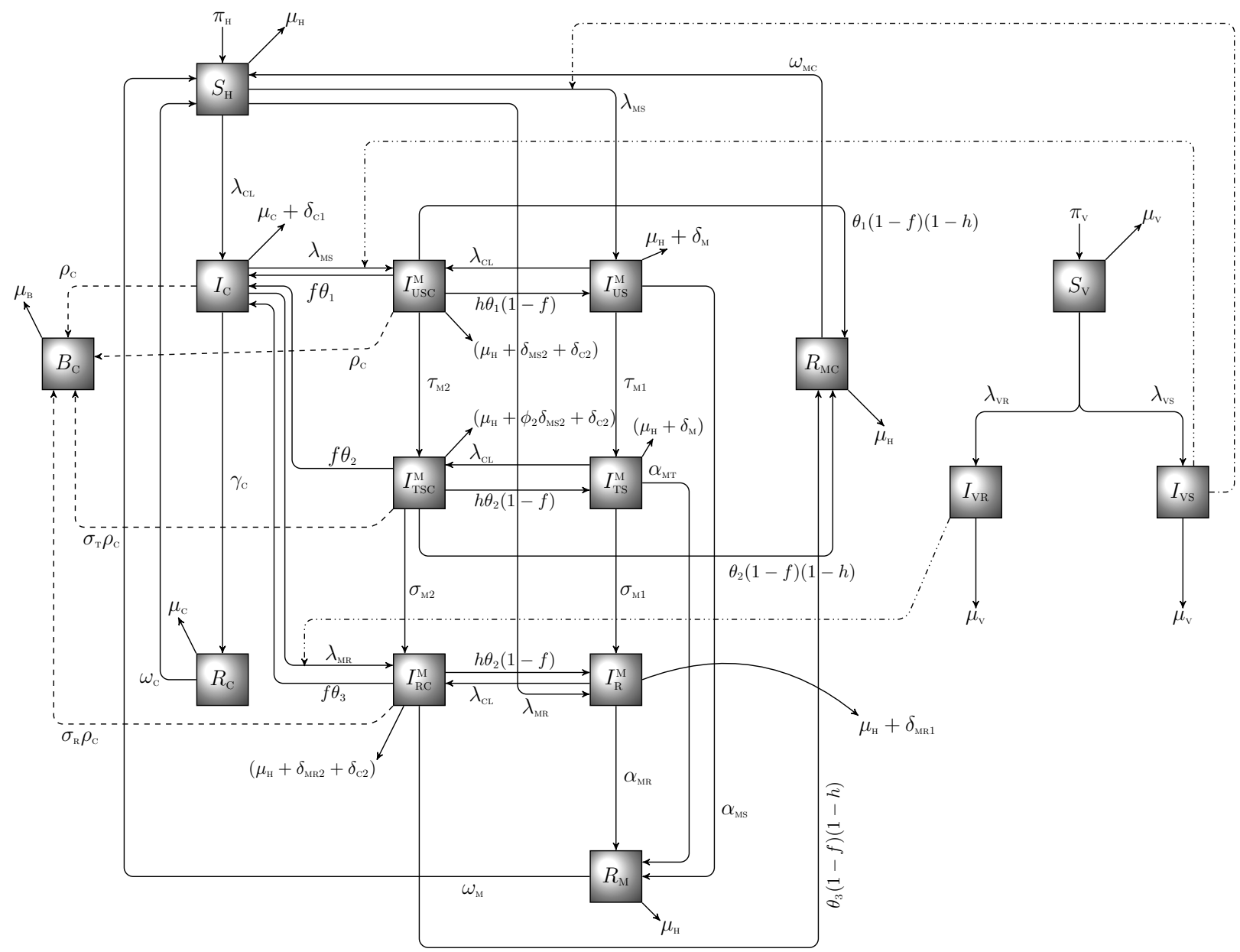

Figure 1: Schematic diagram of the model (2), with $\lambda_{\mathrm{MS}}=\frac{\beta_{\mathrm{M}} b I_{\mathrm{VS}}}{N_{\mathrm{H}}}, \lambda_{\mathrm{MR}}=\frac{\beta_{\mathrm{M}} b \eta_{\mathrm{R}} I_{\mathrm{VR}}}{N_{\mathrm{H}}}$, $\lambda_{\mathrm{CL}}=\frac{B_{\mathrm{C}} \varphi}{\kappa+B_{\mathrm{C}}}, \lambda_{\mathrm{vS}}=\frac{\beta_{\mathrm{v}} b\left[I_{\mathrm{US}}+\eta_{\mathrm{T}}\left(I_{\mathrm{TS}}^{\mathrm{M}}+I_{\mathrm{TSC}}^{\mathrm{M}}\right)\right]}{N_{\mathrm{H}}}, \lambda_{\mathrm{VR}}=\frac{\beta_{\mathrm{V}} b \epsilon_{\mathrm{R}}\left(I_{\mathrm{R}}^{\mathrm{M}}+I_{\mathrm{RC}}^{\mathrm{M}}\right)}{N_{\mathrm{H}}}$ 


$$
\begin{aligned}
& \frac{d S_{\mathrm{H}}}{d t}=\pi_{\mathrm{H}}-\frac{\beta_{\mathrm{M}} b\left(I_{\mathrm{VS}}+\eta_{\mathrm{R}} I_{\mathrm{VR}}\right)}{N_{\mathrm{H}}} S_{\mathrm{H}}-\frac{B_{\mathrm{C}} \varphi}{\kappa+B_{\mathrm{C}}} S_{\mathrm{H}}-\mu_{\mathrm{H}} S_{\mathrm{H}}+\omega_{\mathrm{M}} R_{\mathrm{M}}+\omega_{\mathrm{C}} R_{\mathrm{C}}+\omega_{\mathrm{MC}} R_{\mathrm{MC}} \\
& \frac{d I_{\mathrm{US}}^{\mathrm{M}}}{d t}=\beta_{\mathrm{M}} b \frac{I_{\mathrm{vs}}}{N_{\mathrm{H}}} S_{\mathrm{H}}-\left(\tau_{\mathrm{M} 1}+\mu_{\mathrm{H}}+\alpha_{\mathrm{MS}}+\delta_{\mathrm{MS} 1}\right) I_{\mathrm{Us}}^{\mathrm{M}}-\frac{B_{\mathrm{C}} \varphi}{\kappa+B_{\mathrm{C}}} I_{\mathrm{US}}^{\mathrm{M}}+\frac{\beta_{\mathrm{v}} b I_{\mathrm{vs}}}{N_{\mathrm{H}}} R_{\mathrm{C}}+h \theta_{1}(1-f) I_{\mathrm{USC}}^{\mathrm{M}} \\
& \frac{d I_{\mathrm{TS}}^{\mathrm{M}}}{d t}=\tau_{\mathrm{M} 1} I_{\mathrm{US}}^{\mathrm{M}}-\left(\sigma_{\mathrm{M} 1}+\mu_{\mathrm{H}}+\alpha_{\mathrm{MT}}+\phi_{1} \delta_{\mathrm{MS} 1}\right) I_{\mathrm{TS}}^{\mathrm{M}}-\frac{B_{\mathrm{c}} \varphi}{\kappa+B_{\mathrm{C}}} I_{\mathrm{TS}}^{\mathrm{M}}+h \theta_{2}(1-f) I_{\mathrm{TSC}}^{\mathrm{M}} \\
& \frac{d I_{\mathrm{R}}^{\mathrm{M}}}{d t}=\sigma_{\mathrm{M} 1} I_{\mathrm{TS}}^{\mathrm{M}}+\frac{\beta_{\mathrm{M}} b \eta_{\mathrm{R}} I_{\mathrm{VR}}}{N_{\mathrm{H}}} S_{\mathrm{H}}-\left(\mu_{\mathrm{H}}+\alpha_{\mathrm{MR}}+\delta_{\mathrm{MR} 1}\right) I_{\mathrm{R}}^{\mathrm{M}}-\frac{B_{\mathrm{C}} \varphi}{\kappa+B_{\mathrm{C}}} I_{\mathrm{R}}^{\mathrm{M}}+\frac{\beta_{\mathrm{M}} b \eta_{\mathrm{R}} I_{\mathrm{VR}}}{N_{\mathrm{H}}} R_{\mathrm{C}}+h \theta_{3}(1-f) I_{\mathrm{RC}}^{\mathrm{M}} \\
& \frac{d R_{\mathrm{M}}}{d t}=\alpha_{\mathrm{MS}} I_{\mathrm{US}}^{\mathrm{M}}+\alpha_{\mathrm{MT}} I_{\mathrm{TS}}^{\mathrm{M}}+\alpha_{\mathrm{MR}} I_{\mathrm{R}}^{\mathrm{M}}-\left(\mu+\omega_{\mathrm{M}}\right) R_{\mathrm{M}}-\frac{B_{\mathrm{c}} \varphi}{\kappa+B_{\mathrm{C}}} R_{\mathrm{M}} \\
& \frac{d I_{\mathrm{C}}}{d t}=\frac{\beta_{\mathrm{C}} \varphi}{\kappa+B_{\mathrm{C}}}\left(S_{\mathrm{H}}+R_{\mathrm{M}}\right)-\left(\delta_{\mathrm{C} 1}+\mu_{\mathrm{H}}+\gamma_{\mathrm{C}}\right) I_{\mathrm{C}}-\frac{\beta_{\mathrm{V}} b I_{\mathrm{VS}}}{N_{\mathrm{H}}} I_{\mathrm{C}}-\frac{\beta_{\mathrm{M}} b \eta_{\mathrm{R}} I_{\mathrm{VR}}}{N_{\mathrm{H}}} I_{\mathrm{C}} \\
& +f \theta_{1} I_{\mathrm{USC}}^{\mathrm{M}}+f \theta_{2} I_{\mathrm{TSC}}^{\mathrm{M}}+f \theta_{3} I_{\mathrm{RC}}^{\mathrm{M}} \\
& \frac{d R_{\mathrm{C}}}{d t}=\gamma_{\mathrm{C}} I_{\mathrm{C}}-\left(\mu_{\mathrm{H}}+\omega_{\mathrm{C}}\right) R_{\mathrm{C}}-\frac{\beta_{\mathrm{M}} b\left(I_{\mathrm{vS}}+\eta_{\mathrm{R}} I_{\mathrm{VR}}\right)}{N_{\mathrm{H}}} R_{\mathrm{C}} \\
& \frac{d B_{\mathrm{C}}}{d t}=\rho_{\mathrm{C}} I_{\mathrm{C}}+\rho_{\mathrm{C}}\left(I_{\mathrm{uSC}}^{\mathrm{M}}+\sigma_{\mathrm{T}} I_{\mathrm{TSC}}^{\mathrm{M}}+\sigma_{\mathrm{R}} I_{\mathrm{RC}}^{\mathrm{M}}\right)-\mu_{\mathrm{B}} B_{\mathrm{C}} \\
& \frac{d I_{\mathrm{uSC}}^{\mathrm{M}}}{d t}=\frac{B_{\mathrm{C}} \varphi}{\kappa+B_{\mathrm{C}}} I_{\mathrm{us}}^{\mathrm{M}}+\frac{\beta_{\mathrm{M}} b I_{\mathrm{vs}}}{N_{\mathrm{H}}} I_{\mathrm{C}}-\mu_{\mathrm{H}} I_{\mathrm{usc}}^{\mathrm{M}}-\delta_{\mathrm{Ms} 2} I_{\mathrm{usC}}^{\mathrm{M}}-\delta_{\mathrm{C} 2} I_{\mathrm{usC}}^{\mathrm{M}}-\tau_{\mathrm{M} 2} I_{\mathrm{UsC}}^{\mathrm{M}}-\theta_{1} I_{\mathrm{usC}}^{\mathrm{M}} \\
& \frac{d I_{\mathrm{TSC}}^{\mathrm{M}}}{d t}=\frac{B_{\mathrm{C}} \varphi}{\kappa+B_{\mathrm{C}}} I_{\mathrm{TS}}^{\mathrm{M}}+\tau_{\mathrm{M} 2} I_{\mathrm{UsC}}^{\mathrm{M}}-\mu_{\mathrm{H}} I_{\mathrm{TSC}}^{\mathrm{M}}-\phi_{2} \delta_{\mathrm{MS} 2} I_{\mathrm{TSC}}^{\mathrm{M}}-\theta_{2} I_{\mathrm{TSC}}^{\mathrm{M}}-\sigma_{\mathrm{M} 2} I_{\mathrm{TSC}}^{\mathrm{M}}-\delta_{\mathrm{C} 2} I_{\mathrm{TSC}}^{\mathrm{M}} \\
& \frac{d I_{\mathrm{RC}}^{\mathrm{M}}}{d t}=\sigma_{\mathrm{M} 2} I_{\mathrm{TSC}}^{\mathrm{M}}+\frac{B_{\mathrm{c}} \varphi}{\kappa+B_{\mathrm{C}}} I_{\mathrm{R}}^{\mathrm{M}}+\frac{\beta_{\mathrm{v}} b \eta_{\mathrm{R}} I_{\mathrm{R}}^{\mathrm{M}}}{N_{\mathrm{H}}} I_{\mathrm{C}}-\mu_{\mathrm{H}} I_{\mathrm{RC}}^{\mathrm{M}}-\delta_{\mathrm{MR} 2} I_{\mathrm{RC}}^{\mathrm{M}}-\delta_{\mathrm{C} 2} I_{\mathrm{RC}}^{\mathrm{M}}-\theta_{3} I_{\mathrm{RC}}^{\mathrm{M}} \\
& \frac{d R_{\mathrm{MC}}}{d t}=\theta_{1}(1-f)(1-h) I_{\mathrm{UsC}}^{\mathrm{M}}+\theta_{2}(1-f)(1-h) I_{\mathrm{TSC}}^{\mathrm{M}}+\theta_{3}(1-f)(1-h) I_{\mathrm{RC}}^{\mathrm{M}}-\left(\omega_{\mathrm{MC}}+\mu_{\mathrm{H}}\right) R_{\mathrm{MC}} \\
& \frac{d S_{\mathrm{v}}}{d t}=\pi_{\mathrm{v}}-\frac{\beta_{\mathrm{v}} b\left(I_{\mathrm{US}}^{\mathrm{M}}+\eta_{\mathrm{T}} I_{\mathrm{TS}}^{\mathrm{M}}+\eta_{\mathrm{R}} I_{\mathrm{R}}^{\mathrm{M}}\right)}{N_{\mathrm{H}}} S_{\mathrm{v}}-\frac{\beta_{\mathrm{v}} b\left(I_{\mathrm{USC}}^{\mathrm{M}}+\eta_{\mathrm{T}} I_{\mathrm{TSC}}^{\mathrm{M}}+\eta_{\mathrm{R}} I_{\mathrm{RC}}^{\mathrm{M}}\right)}{N_{\mathrm{H}}} S_{\mathrm{v}}-\mu_{\mathrm{v}} S_{\mathrm{v}} \\
& \frac{d I_{\mathrm{vS}}}{d t}=\frac{\beta_{\mathrm{v}} b\left(I_{\mathrm{US}}^{\mathrm{M}}+\eta_{\mathrm{T}} I_{\mathrm{TS}}^{\mathrm{M}}\right)}{N_{\mathrm{H}}} S_{\mathrm{v}}+\frac{\beta_{\mathrm{v}} b\left(I_{\mathrm{USC}}^{\mathrm{M}}+\eta_{\mathrm{T}} I_{\mathrm{TSC}}^{\mathrm{M}}\right)}{N_{\mathrm{H}}} S_{\mathrm{v}}-\mu_{\mathrm{v}} I_{\mathrm{vS}} \\
& \frac{d I_{\mathrm{VR}}}{d t}=\frac{\beta_{\mathrm{v}} b \eta_{\mathrm{R}} I_{\mathrm{R}}^{\mathrm{M}}}{N_{\mathrm{H}}} S_{\mathrm{V}}+\frac{\beta_{\mathrm{v}} b \eta_{\mathrm{R}} I_{\mathrm{RC}}^{\mathrm{M}}}{N_{\mathrm{H}}} S_{\mathrm{V}}-\mu_{\mathrm{v}} I_{\mathrm{VR}} \\
& G=\left(S_{\mathrm{H}}, I_{\mathrm{US}}^{\mathrm{M}}, I_{\mathrm{R}}^{\mathrm{M}},,_{\mathrm{C}}, R_{\mathrm{C}}, I_{\mathrm{TSC}}, I_{\mathrm{UsC}}^{\mathrm{M}}, R_{\mathrm{MC}}, I_{\mathrm{RC}}^{\mathrm{M}}\right) \epsilon \Re_{+}^{10}: N \leqslant \frac{\pi}{\mu_{\mathrm{H}}}
\end{aligned}
$$

\section{Mathematical analysis of the co-infection model}

In this section, we qualitatively analyze the model (2) to better understand the dynamics of the coinfection of drug-resistant Malaria and cholera in a population.

\subsection{Basic properties of the Malaria-Cholera co-infection model}

\subsection{Positivity and boundedness of solutions}

For the model (2) to be epidemiologically meaningful, it is important to prove that all its state variables are non-negative for all time $(t)$. In other words, solutions of the model system (2) with positive initial data will remain positive for all time $t>0$.

Theorem 3.1 Let the initial data $S_{\mathrm{H}}>0, I_{\mathrm{US}}^{\mathrm{M}}>0, I_{\mathrm{TS}}^{\mathrm{M}}>0, I_{\mathrm{R}}^{\mathrm{M}}>0, R_{\mathrm{M}}>0, I_{\mathrm{C}}>0, R_{\mathrm{C}}>0, B_{\mathrm{C}}>0, I_{\mathrm{Usc}}^{\mathrm{M}}>$ $0, I_{\mathrm{TSC}}^{\mathrm{M}}>0, I_{\mathrm{RC}}^{\mathrm{M}}>0, R_{\mathrm{MC}}>0, S_{\mathrm{v}}>0, I_{\mathrm{vS}}>0, I_{\mathrm{VR}}>0$. Then the solution of the model are positive for all $t>0$ 
medRxiv preprint doi: https://doi.org/10.1101/2020.08.18.20177329; this version posted September 10, 2020. The copyright holder for this preprint (which was not certified by peer review) is the author/funder, who has granted medRxiv a license to display the preprint in

It is made available under a CC-BY-NC-ND 4.0 International license .

Proof. Let

$t_{1}=\sup \left\{t>0: S_{\mathrm{H}}>0, I_{\mathrm{US}}^{\mathrm{M}}>0, I_{\mathrm{TS}}^{\mathrm{M}}>0, I_{\mathrm{R}}^{\mathrm{M}}>0, R_{\mathrm{M}}>0, I_{\mathrm{C}}>0, R_{\mathrm{C}}>0, B_{\mathrm{C}}>0, I_{\mathrm{USC}}^{\mathrm{M}}>0, I_{\mathrm{TSC}}^{\mathrm{M}}>0, I_{\mathrm{RC}}^{\mathrm{M}}>\right.$ $\left.0, R_{\mathrm{MC}}>0, S_{\mathrm{v}}>0, I_{\mathrm{VS}}>0, I_{\mathrm{VR}}>0 \in[0, t]\right\}$. Thus, $t_{1}>0$.

We have, from the first equation of the system (2) that

$$
\begin{aligned}
& \frac{d S_{\mathrm{H}}}{d t}=\pi_{\mathrm{H}}-\left(\lambda_{\mathrm{VS}}+\lambda_{\mathrm{VR}}+\lambda_{\mathrm{CL}}+\mu_{\mathrm{H}}\right) S_{\mathrm{H}}+\omega_{\mathrm{M}} R_{\mathrm{M}}+\omega_{\mathrm{C}} R_{\mathrm{C}}+\omega_{\mathrm{MC}} R_{\mathrm{MC}} \\
& \frac{d S_{\mathrm{H}}}{d t}>\pi_{\mathrm{H}}-\left(\lambda_{\mathrm{VS}}+\lambda_{\mathrm{VR}}+\lambda_{\mathrm{CL}}+\mu_{\mathrm{H}}\right) S_{\mathrm{H}}
\end{aligned}
$$

which can be re-written as

$$
\frac{d}{d t}\left\{S_{\mathrm{H}}(t) \exp \left[\int_{0}^{t}\left(\lambda_{\mathrm{VS}}(u)+\lambda_{\mathrm{VR}}(u)\right) d u+\lambda_{\mathrm{CL}}(u)+\mu_{\mathrm{H}}(t)\right]\right\}>\pi_{\mathrm{H}} \exp \left[\int_{0}^{t}\left(\lambda_{\mathrm{VS}}(u)+\lambda_{\mathrm{VR}}(u) d u+\lambda_{\mathrm{CL}}(u) d u\right)+\mu_{\mathrm{H}}(t)\right]
$$

Hence:

$\frac{d}{d t}\left\{S_{\mathrm{H}}\left(t_{1}\right) \exp \left[\int_{0}^{t_{1}}\left(\lambda_{\mathrm{VS}}(u)+\lambda_{\mathrm{VR}}(u)+\lambda_{\mathrm{CL}}(u)\right) d u+\mu_{\mathrm{H}}(t)_{1}\right]\right\}-S_{\mathrm{H}}(0)>\pi_{\mathrm{H}} \int_{0}^{t_{1}} \exp \left[\int_{0}^{x}\left(\lambda_{\mathrm{VS}}(u)+\lambda_{\mathrm{VR}}(u)+\lambda_{\mathrm{CL}}(u)\right) d u+\mu_{\mathrm{H}}(x)\right] d x$

so that

$$
\begin{aligned}
S_{\mathrm{H}}\left(t_{1}\right) & >S_{\mathrm{H}}(0) \exp \left[-\int_{0}^{t_{1}}\left(\lambda_{\mathrm{VS}}(u)+\lambda_{\mathrm{VR}}(u)+\lambda_{\mathrm{CL}}(u)\right) d u-\mu_{\mathrm{H}} t_{1}\right]+\exp \left[-\int_{0}^{t_{1}}\left(\lambda_{\mathrm{VS}}(u)+\lambda_{\mathrm{VR}}(u)+\lambda_{\mathrm{CL}}(u)\right) d u-\mu_{\mathrm{H}} t_{1}\right] \\
& \times \Pi_{\mathrm{M}} \int_{0}^{t_{1}} \exp \left[\int_{0}^{x}\left(\lambda_{\mathrm{VS}}(u)+\lambda_{\mathrm{VR}}(u)+\lambda_{\mathrm{CL}}(u)\right) d u+\mu_{\mathrm{H}}(x)\right] d x>0
\end{aligned}
$$

Similarly, it can be shown that:

$I_{\mathrm{US}}^{\mathrm{M}}>0, I_{\mathrm{TS}}^{\mathrm{M}}>0, I_{\mathrm{R}}^{\mathrm{M}}>0, R_{\mathrm{M}}>0, I_{\mathrm{C}}>0, R_{\mathrm{C}}>0, B_{\mathrm{C}}>0, I_{\mathrm{USC}}^{\mathrm{M}}>0, I_{\mathrm{TSC}}^{\mathrm{M}}>0, I_{\mathrm{RC}}^{\mathrm{M}}>0, R_{\mathrm{MC}}>0, S_{\mathrm{v}}>0, I_{\mathrm{vS}}>$ $0, I_{\mathrm{VR}}>0$.

\subsection{Invariant regions}

The co-infection model (2) will be analyzed in a biologically feasible region as follows. We first show that the system (4) is dissipative in a proper subset $\mathcal{D} \subset \mathfrak{R}_{+}^{15}$. The system (2) is split into two parts, namely the human population $\left(N_{\mathrm{H}}\right)$ (with $\left.N_{\mathrm{H}}=S_{\mathrm{H}}+I_{\mathrm{US}}^{\mathrm{M}}+I_{\mathrm{TS}}^{\mathrm{M}}+I_{\mathrm{R}}^{\mathrm{M}}+R_{\mathrm{M}}+I_{\mathrm{C}}+R_{\mathrm{C}}+I_{\mathrm{USC}}^{\mathrm{M}}+I_{\mathrm{TSC}}^{\mathrm{M}}+I_{\mathrm{RC}}^{\mathrm{M}}+R_{\mathrm{MC}}\right)$, the bacteria population, $B_{\mathrm{C}}$ and the vector population, $\left(N_{\mathrm{v}}\right)$ (with $\left.N_{\mathrm{v}}=S_{\mathrm{v}}+I_{\mathrm{Vs}}+I_{\mathrm{VR}}\right)$.

$$
\begin{aligned}
\mathcal{D}_{\mathrm{H}}= & \left\{\left(S_{\mathrm{H}}, I_{\mathrm{US}}^{\mathrm{M}}, I_{\mathrm{TS}}^{\mathrm{M}}, I_{\mathrm{R}}^{\mathrm{M}}, R_{\mathrm{M}}, I_{\mathrm{C}}, R_{\mathrm{C}}, I_{\mathrm{USC}}^{\mathrm{M}}, I_{\mathrm{TSC}}^{\mathrm{M}}, I_{\mathrm{RC}}^{\mathrm{M}}, R_{\mathrm{MC}}\right):\right. \\
& \left.S_{\mathrm{H}}+I_{\mathrm{US}}^{\mathrm{M}}+I_{\mathrm{TS}}^{\mathrm{M}}+I_{\mathrm{R}}^{\mathrm{M}}+R_{\mathrm{M}}+I_{\mathrm{C}}+R_{\mathrm{C}}+I_{\mathrm{USC}}^{\mathrm{M}}+I_{\mathrm{TSC}}^{\mathrm{M}}+I_{\mathrm{RC}}^{\mathrm{M}}+R_{\mathrm{MC}} \leq \frac{\pi_{\mathrm{H}}}{\mu_{\mathrm{H}}}\right\}, \\
\mathcal{D}_{\mathrm{B}}= & \left\{B_{\mathrm{C}}: B_{\mathrm{C}} \leq \frac{\left(2 \rho_{\mathrm{C}}+\rho_{\mathrm{C}} \sigma_{\mathrm{T}}+\rho_{\mathrm{C}} \sigma_{\mathrm{R}}\right) \pi_{\mathrm{H}}}{\mu_{\mathrm{H}} \mu_{\mathrm{B}}}\right\} \\
\mathcal{D}_{\mathrm{v}}= & \left\{\left(S_{\mathrm{v}}, I_{\mathrm{vs}}, I_{\mathrm{MVR}}\right):: S_{\mathrm{v}}+I_{\mathrm{vS}}+I_{\mathrm{vR}} \leq \frac{\pi_{\mathrm{v}}}{\mu_{\mathrm{v}}}\right\}
\end{aligned}
$$

The following steps are followed to establish the positive invariance of $\mathcal{D}$ (i.e. solutions in $\mathcal{D}$ remain in $\mathcal{D}$ for all time $t>0)$.

Adding human, bacteria and vector components of the differential system (2), respectively, gives

$$
\begin{aligned}
\frac{d N_{\mathrm{H}}}{d t}= & \pi_{\mathrm{H}}-\mu_{\mathrm{H}} N_{\mathrm{H}}(t)-\left[\delta_{\mathrm{MS} 1} I_{\mathrm{US}}^{\mathrm{M}}+\phi_{1} \delta_{\mathrm{MS} 1} I_{\mathrm{TS}}^{\mathrm{M}}+\delta_{\mathrm{MR} 1} I_{\mathrm{R}}^{\mathrm{M}}+\delta_{\mathrm{C} 1} I_{\mathrm{C}}-\delta_{\mathrm{MS} 2} I_{\mathrm{USC}}^{\mathrm{M}}-\delta_{\mathrm{C} 2} I_{\mathrm{USC}}^{\mathrm{M}}\right. \\
& \left.-\phi_{2} \delta_{\mathrm{MS} 2} I_{\mathrm{TSC}}^{\mathrm{M}}-\delta_{\mathrm{MR} 2} I_{\mathrm{RC}}^{\mathrm{M}}-\delta_{\mathrm{C} 2} I_{\mathrm{RC}}^{\mathrm{M}}\right] \\
\frac{d B_{\mathrm{C}}}{d t}= & \rho_{\mathrm{C}} I_{\mathrm{C}}+\rho_{\mathrm{C}}\left(I_{\mathrm{USC}}^{\mathrm{M}}+\sigma_{\mathrm{T}} I_{\mathrm{TSC}}^{\mathrm{M}}+\sigma_{\mathrm{R}} I_{\mathrm{RC}}^{\mathrm{M}}\right)-\mu_{\mathrm{B}} B_{\mathrm{C}}(t) \\
\frac{d N_{\mathrm{v}}}{d t}= & \pi_{\mathrm{v}}-\mu_{\mathrm{v}} N_{\mathrm{v}}(t)
\end{aligned}
$$


medRxiv preprint doi: https://doi.org/10.1101/2020.08.18.20177329; this version posted September 10, 2020. The copyright holder for this preprint (which was not certified by peer review) is the author/funder, who has granted medRxiv a license to display the preprint in

From (3), we have that

$$
\begin{aligned}
& \pi_{\text {н }}-\left(\mu+9 \delta_{\text {н }}\right) N_{\text {н }} \leq \frac{d N_{\text {н }}}{d t}<\pi_{\text {н }}-\mu_{\text {Н }} N_{\text {н }} \\
& \pi_{\text {н }}-\left(\mu+9 \delta_{\text {н }}\right) N_{\text {н }} \leq \frac{d N_{\text {н }}}{d t}<\pi_{\text {н }}-\mu_{\text {н }} N_{\text {н }} \\
& \frac{d N_{\mathrm{v}}}{d t} \leq \pi_{\mathrm{v}}-\mu_{\mathrm{v}} N_{\mathrm{v}}
\end{aligned}
$$

where $\delta_{\mathrm{H}}=\min \left\{\delta_{\mathrm{MS} 1}, \phi_{1} \delta_{\mathrm{MS} 1}, \delta_{\mathrm{MR} 1}, \delta_{\mathrm{C} 1}, \delta_{\mathrm{MS} 2}, \delta_{\mathrm{C} 2}, \phi_{2} \delta_{\mathrm{MS} 2}, \delta_{\mathrm{MR} 2}\right\}$

Using the Comparison theorem [19], we have that, $N_{\mathrm{H}}(t) \leq \frac{\pi_{\mathrm{H}}}{\mu_{\mathrm{H}}}$ and $N_{\mathrm{V}}(t) \leq \frac{\pi_{\mathrm{V}}}{\mu_{\mathrm{V}}}$ if $N_{\mathrm{H}}(0) \leq \frac{\pi_{\mathrm{H}}}{\mu_{\mathrm{H}}}$ and $N_{\mathrm{v}}(0) \leq \frac{\pi_{\mathrm{v}}}{\mu_{\mathrm{v}}}$ respectively. Thus, the region $\mathcal{D}$ is positively invariant. Hence, it is sufficient to consider the dynamics of the flow generated by the system (2) in $\mathcal{D}$. In this region, the model can be considered as being epidemiologically and mathematically well-posed [20]. Thus, every solution of the model (2) with initial conditions in $\mathcal{D}$ remains in $\mathcal{D}$ for all time $t \geq 0$. Therefore, the $\omega$-limit sets of the system (2) are contained in $\mathcal{D}$. Thus result is summarized thus.

Lemma 3.1 The region $\mathcal{D}=\mathcal{D}_{\mathrm{H}} \cup \mathcal{D}_{\mathrm{B}} \cup \mathcal{D}_{\mathrm{v}} \subset \mathfrak{R}_{+}^{11} \times \mathfrak{R}_{+} \times \mathfrak{R}_{+}^{3}$ is positively-invariant for the model (2) with initial conditions in $\mathfrak{R}_{+}^{15}$.

\subsection{Basic reproduction number of the co-infection model (2)}

The Malaria-Cholera co-infection model (2) has a DFE, obtained by setting the right-hand sides of the equations in the model (2) to zero, given by

$$
\begin{aligned}
\xi_{0} & =\left(S_{\mathrm{H}}^{*}, I_{\mathrm{Us}}^{\mathrm{N}^{*}}, I_{\mathrm{TS}}^{\mathrm{M}^{*}}, I_{\mathrm{R}}^{\mathrm{M}^{*}}, R_{\mathrm{M}}^{*}, I_{\mathrm{C}}^{*}, R_{\mathrm{C}}^{*}, B_{\mathrm{C}}^{*}, I_{\mathrm{Usc}}^{\mathrm{N}^{*}}, I_{\mathrm{Tsc}}^{\mathrm{M}^{*}}, I_{\mathrm{RC}}^{\mathrm{M}^{*}}, R_{\mathrm{MC}}^{*}, S_{\mathrm{v}}^{*}, I_{\mathrm{vs}}^{*}, I_{\mathrm{vR}}^{*}\right) \\
& =\left(\frac{\pi_{\mathrm{H}}}{\mu_{\mathrm{H}}}, 0,0,0,0,0,0,0,0,0,0,0, \frac{\pi_{\mathrm{v}}}{\mu_{\mathrm{v}}}, 0,0\right)
\end{aligned}
$$

The matrices $F$ and $V$, for the new infection terms and the remaining transfer terms, evaluated at the disease free equilibrium (DFE) are, respectively, given by

$$
\begin{gathered}
F=\left(\begin{array}{ccccccccccc}
0 & 0 & & 0 & 0 & 0 & 0 & 0 & 0 & \beta_{\mathrm{M}} b & 0 \\
0 & 0 & & 0 & 0 & 0 & 0 & 0 & 0 & 0 & 0 \\
0 & 0 & & 0 & 0 & 0 & 0 & 0 & 0 & 0 & \beta_{\mathrm{M}} b \eta_{\mathrm{R}} \\
0 & 0 & & 0 & 0 & \frac{\varphi S_{\mathrm{H}}}{K} & 0 & 0 & 0 & 0 & 0 \\
0 & 0 & 0 & 0 & 0 & 0 & 0 & 0 & 0 & 0 \\
0 & 0 & 0 & 0 & 0 & 0 & 0 & 0 & 0 & 0 \\
0 & 0 & 0 & 0 & 0 & 0 & 0 & 0 & 0 & 0 \\
0 & 0 & 0 & 0 & 0 & 0 & 0 & 0 & 0 & 0 \\
\frac{\beta_{\mathrm{V}} b S_{\mathrm{V}}}{N_{\mathrm{H}}} & \frac{\eta_{\mathrm{T}} \beta_{\mathrm{V}} b S_{\mathrm{V}}}{N_{\mathrm{H}}} & 0 & 0 & 0 & \frac{\beta_{\mathrm{V}} b S_{\mathrm{V}}}{N_{\mathrm{H}}} & \frac{\eta_{\mathrm{T}} \beta_{\mathrm{V}} b S_{\mathrm{V}}}{N_{\mathrm{H}}} & 0 & 0 & 0 \\
0 & 0 & \frac{\beta_{\mathrm{V}} b \eta_{\mathrm{R}} S_{\mathrm{V}}}{N_{\mathrm{H}}} & 0 & 0 & 0 & 0 & \frac{\beta_{\mathrm{V}} b \eta_{\mathrm{R}} S_{\mathrm{V}}}{N_{\mathrm{H}}} & 0 & 0
\end{array}\right) \\
V=\left(\begin{array}{ccccccccccc}
H_{1} & 0 & 0 & 0 & 0 & G_{1} & 0 & 0 & 0 & 0 \\
\tau_{\mathrm{M} 1} & H_{2} & 0 & 0 & 0 & 0 & G_{2} & 0 & 0 & 0 \\
0 & -\sigma_{\mathrm{M} 1} & H_{3} & 0 & 0 & 0 & 0 & G_{3} & 0 & 0 \\
0 & 0 & 0 & H_{4} & 0 & -f \theta_{1} & f \theta_{2} & -f \theta_{3} & 0 & 0 \\
0 & 0 & 0 & -\rho_{\mathrm{C}} & \mu & -\rho_{\mathrm{C}} & \rho_{\mathrm{C}} \sigma_{\mathrm{T}} & \rho_{\mathrm{C}} \sigma_{\mathrm{R}} & 0 & 0 \\
0 & 0 & 0 & 0 & 0 & H_{5} & 0 & 0 & 0 & 0 \\
0 & 0 & 0 & 0 & 0 & 0 & H_{6} & 0 & 0 & 0 \\
0 & 0 & 0 & 0 & 0 & 0 & \sigma_{\mathrm{M} 1} & H_{7} & 0 & 0 \\
0 & 0 & 0 & 0 & 0 & 0 & 0 & 0 & H_{8} & 0 \\
0 & 0 & 0 & 0 & 0 & 0 & 0 & 0 & 0 & H_{9}
\end{array}\right)
\end{gathered}
$$

where

$$
\begin{aligned}
& H_{1}=\tau_{\mathrm{M} 1}+\mu_{\mathrm{H}}+\alpha_{\mathrm{MS}}+\delta_{\mathrm{MS} 1}, \quad H_{2}=\sigma_{\mathrm{M} 1}+\mu_{\mathrm{H}}+\alpha_{\mathrm{MT}}+\phi_{1} \delta_{\mathrm{M} 1}, \quad H_{3}=\mu_{\mathrm{H}}+\alpha_{\mathrm{MR}}+\delta_{\mathrm{MR} 1}, \quad H_{4}=\delta_{\mathrm{C} 1}+\mu_{\mathrm{H}}+\gamma_{\mathrm{C}} \\
& H_{5}=\mu_{\mathrm{H}}+\delta_{\mathrm{MS} 2}+\delta_{\mathrm{C} 2}+\tau_{\mathrm{M} 2}+\theta_{1}, \quad H_{6}=\phi_{1} \delta_{\mathrm{MS} 2}+\mu_{\mathrm{H}}+\theta_{2} \sigma_{\mathrm{M} 2}+\delta_{\mathrm{C} 2}, \quad H_{7}=\delta_{\mathrm{MR} 2}+\mu_{\mathrm{H}}+\delta_{\mathrm{C} 2}+\theta_{3}
\end{aligned}
$$


medRxiv preprint doi: https://doi.org/10.1101/2020.08.18.20177329; this version posted September 10, 2020. The copyright holder for this preprint (which was not certified by peer review) is the author/funder, who has granted medRxiv a license to display the preprint in

It is made available under a CC-BY-NC-ND 4.0 International license .

The basic reproduction number of the Malaria-Cholera co-infection model (2), using the approach illustrated in van den Driessche and Watmough [21, is given by $\mathcal{R}_{0}=\max \left\{\mathcal{R}_{0 \mathrm{HP}}, \mathcal{R}_{0 \mathrm{CL}}\right\}$ where $\mathcal{R}_{0 \mathrm{M}}$ and $\mathcal{R}_{0 \mathrm{c}}$ are, respectively, the Malaria and Cholera associated reproduction numbers, given by

$$
\begin{gathered}
\mathcal{R}_{0 \mathrm{MS}}=\sqrt{\frac{b^{2} \beta_{\mathrm{M}} \beta_{\mathrm{v}}\left(H_{2}+\eta_{\mathrm{T}} \tau_{\mathrm{M} 1}\right) S_{\mathrm{v}}^{*}}{H_{1} H_{2} \mu_{\mathrm{v}} N_{\mathrm{H}}^{*}}} \\
\mathcal{R}_{0 \mathrm{MR}}=\sqrt{\frac{b^{2} \eta_{\mathrm{R}}^{2} \beta_{\mathrm{M}} \beta_{\mathrm{v}} S_{\mathrm{v}}^{*}}{H_{3} \mu_{\mathrm{v}} N_{\mathrm{H}}^{*}}}
\end{gathered}
$$

and

$$
\mathcal{R}_{0 \mathrm{CL}}=\frac{\varphi S_{\mathrm{H}}^{*} \rho_{\mathrm{C}}}{\mu_{\mathrm{B}} \kappa H_{4}}
$$

\subsection{Local asymptotic stability of disease-free equilibrium (DFE) of the co-infection model (2)}

Lemma 3.2 The DFE, $\xi_{0}$, of the Malaria-Cholera co-infection model (2) is locally asymptotically stable if $\mathcal{R}_{0}<1$, and unstable if $\mathcal{R}_{0}>1$.

Proof

The local stability of the co-infection model is analysed by the Jacobian matrix of the system (2) at $\xi_{0}$, given by:

$J\left(\xi_{0}\right)=\left(\begin{array}{cccccccccccccccc}-\mu_{\mathrm{H}} & 0 & 0 & 0 & \omega_{\mathrm{M}} & 0 & \omega_{\mathrm{C}} & -\frac{\varphi S_{\mathrm{H}}^{*}}{\kappa} & 0 & 0 & 0 & \omega_{\mathrm{MC}} & 0 & -\beta_{\mathrm{M}} b & -\beta_{\mathrm{M}} \eta_{\mathrm{R}} b \\ 0 & -H_{1} & 0 & 0 & 0 & 0 & 0 & 0 & G_{1} & 0 & 0 & 0 & 0 & \beta_{\mathrm{M}} b & 0 \\ 0 & \tau_{\mathrm{M} 1} & -H_{2} & 0 & 0 & 0 & 0 & 0 & 0 & G_{2} & 0 & 0 & 0 & 0 & 0 \\ 0 & 0 & \sigma_{\mathrm{M} 1} & -H_{3} & 0 & 0 & 0 & 0 & 0 & 0 & G_{3} & 0 & 0 & 0 & 0 \\ \alpha_{\mathrm{M} 2} & \alpha_{\mathrm{MT}} & \alpha_{\mathrm{MR}} & -D_{1} & 0 & 0 & 0 & 0 & 0 & 0 & 0 & 0 & 0 & 0 & 0 \\ 0 & 0 & 0 & 0 & 0 & -H_{4} & 0 & \frac{\varphi S_{\mathrm{H}}^{*}}{\kappa} & f \theta_{1} & f \theta_{2} & f \theta_{3} & 0 & 0 & 0 & 0 \\ 0 & 0 & 0 & 0 & 0 & \gamma_{\mathrm{C}} & -D_{2} & 0 & 0 & 0 & 0 & 0 & 0 & 0 & 0 \\ 0 & 0 & 0 & 0 & 0 & \rho_{\mathrm{C}} & 0 & -\mu_{\mathrm{B}} & \rho_{\mathrm{C}} & \rho_{\mathrm{C}} \sigma_{\mathrm{T}} & \rho_{\mathrm{C}} \sigma_{\mathrm{R}} & 0 & 0 & 0 & 0 \\ 0 & 0 & 0 & 0 & 0 & 0 & 0 & 0 & -H_{5} & 0 & 0 & 0 & 0 & 0 & 0 \\ 0 & 0 & 0 & 0 & 0 & 0 & 0 & 0 & \tau_{\mathrm{M} 2} & -H_{6} & 0 & 0 & 0 & 0 & 0 \\ 0 & 0 & 0 & 0 & 0 & 0 & 0 & 0 & 0 & \sigma_{\mathrm{M} 2} & -H_{7} & 0 & 0 & 0 & 0 \\ 0 & 0 & 0 & 0 & 0 & 0 & 0 & 0 & G_{4} & G_{5} & G_{6} & -D_{3} & 0 & 0 & 0 \\ 0 & -\Phi & -\Phi \eta_{\mathrm{T}} & -\Phi \eta_{\mathrm{R}} & 0 & 0 & 0 & 0 & -\Phi & -\Phi \eta_{\mathrm{T}} & -\Phi \eta_{\mathrm{R}} & 0 & -\mu_{\mathrm{V}} & 0 & 0 \\ 0 & \Phi & \Phi \eta_{\mathrm{T}} & 0 & 0 & 0 & 0 & 0 & \Phi & \Phi \eta_{\mathrm{T}} & 0 & 0 & 0 & -\mu_{\mathrm{V}} & 0 \\ 0 & 0 & 0 & \Phi \eta_{\mathrm{R}} & 0 & 0 & 0 & 0 & 0 & 0 & \Phi \eta_{\mathrm{R}} & 0 & 0 & 0 & -\mu_{\mathrm{V}}\end{array}\right)$

with,

$H_{1}=\tau_{\mathrm{M} 1}+\mu_{\mathrm{H}}+\alpha_{\mathrm{MS}}+\delta_{\mathrm{MS} 1}, \quad H_{2}=\sigma_{\mathrm{M} 1}+\mu_{\mathrm{H}}+\alpha_{\mathrm{MT}}+\phi_{1} \delta_{\mathrm{MS} 1}, \quad H_{3}=\mu_{\mathrm{H}}+\alpha_{\mathrm{MR}}+\delta_{\mathrm{MR} 1}, \quad H_{4}=\delta_{\mathrm{C} 1}+\mu_{\mathrm{H}}+\gamma_{\mathrm{C}}$

$H_{5}=\mu_{\mathrm{H}}+\delta_{\mathrm{MS} 2}+\delta_{\mathrm{C} 2}+\tau_{\mathrm{M} 2}+\theta_{1}, \quad H_{6}=\phi_{1} \delta_{\mathrm{MS} 2}+\mu_{\mathrm{H}}+\theta_{2} \sigma_{\mathrm{M} 2}+\delta_{\mathrm{C} 2}, \quad H_{7}=\delta_{\mathrm{MR} 2}+\mu_{\mathrm{H}}+\delta_{\mathrm{C} 2}+\theta_{3}$

$G_{1}=h \theta_{1}(1-f), \quad G_{2}=h \theta_{2}(1-f), \quad G_{3}=h \theta_{3}(1-f), \quad G_{4}=\theta_{1}(1-f)(1-h), \quad G_{5}=\theta_{2}(1-f)(1-h)$

$G_{6}=\theta_{3}(1-f)(1-h), \quad D_{1}=\mu_{\mathrm{H}}+\omega_{\mathrm{M}}, \quad D_{2}=\mu_{\mathrm{H}}+\omega_{\mathrm{C}}, \quad D_{3}=\mu_{\mathrm{H}}+\omega_{\mathrm{MC}}, \quad \Phi=\frac{\beta_{\mathrm{v}} b S_{\mathrm{V}}^{*}}{N_{\mathrm{H}}^{*}}$

The eigenvalues are $\lambda_{1}=-D_{1}, \lambda_{1}=-D_{2}, \lambda_{3}=-D_{3}, \lambda_{4}=-H_{5}, \lambda_{5}=-H_{6}, \lambda_{6}=-H_{7}, \lambda_{7}=-\mu_{\mathrm{H}}$, $\lambda_{8}=-\mu_{\mathrm{v}}$ and the solutions of the characteristic polynomials:

$$
\begin{gathered}
\lambda^{2}+\left(H_{3}+\mu_{\mathrm{v}}\right) \lambda+\mu_{\mathrm{v}} H_{3}\left(1-\mathcal{R}_{0 \mathrm{MR}}^{2}\right)=0, \\
\lambda^{3}+\left(H_{1}+H_{2}+\mu_{\mathrm{v}}\right) \lambda^{2}+\left(H_{1} H_{2}+\mu_{\mathrm{v}} H_{1}+\mu_{\mathrm{v}} H_{2}-\frac{b^{2} \beta_{\mathrm{M}} \beta_{\mathrm{v}} S_{\mathrm{v}}^{*}}{N_{\mathrm{H}}^{*}}\right) \lambda+\mu_{\mathrm{v}} H_{1} H_{2}\left(1-\mathcal{R}_{0 \mathrm{MT}}^{2}\right),
\end{gathered}
$$

and

$$
\lambda^{2}+\left(\mu_{\mathrm{B}}+H_{4}\right) \lambda+\mu_{\mathrm{B}} H_{4}\left(1-\mathcal{R}_{0 \mathrm{CL}}\right)
$$

Applying the Routh-Hurwitz criterion, the quadratic equations (5), (6) and (7) will have roots with negative real parts if and only if $\mathcal{R}_{0}<1$. As a result, the disease-free equilibrium, $\xi_{0}$ is locally asymptotically stable if $\mathcal{R}_{0}<1$. 
medRxiv preprint doi: https://doi.org/10.1101/2020.08.18.20177329; this version posted September 10, 2020. The copyright holder for this preprint (which was not certified by peer review) is the author/funder, who has granted medRxiv a license to display the preprint in

It is made available under a CC-BY-NC-ND 4.0 International license .

\subsection{Global asymptotic stability(GAS) of the disease-free equilibrium(DFE) $\xi_{0}$ of the co-infection model (2)}

The approach illustrated in [22] is used to investigate the global asymptotic stability of the disease free equilibrium of the co-infection model. In this section, we list two conditions that if met, also guarantee the global asymptotic stability of the disease-free state. First, system (2) must be written in the form:

$$
\begin{aligned}
& \frac{d X}{d t}=F(X, I) \\
& \frac{d I}{d t}=G(X, I), G(X, 0)=0
\end{aligned}
$$

where $X \in R^{m}$ denotes (its components) the number of uninfected individuals and $I \in R^{n}$ denotes (its components) the number of infected individuals including latent, infectious, etc. $U_{0}=\left(X^{*}, 0\right)$ denotes the disease-free equilibrium of this system. The conditions $(H 1)$ and $(H 2)$ below must be met to guarantee local asymptotic stability:

$(H 1)$ : For $\frac{d X}{d t}=F(X, 0), X^{*}$ is globally asymptotically stable (GAS),

$(H 2): G(X, I)=A I-\hat{G}(X, I) X, G(X, I) \geq 0$ for $(X, I) \in \Omega$,

where $A=D_{1} G\left(X^{*}, 0\right)$ is an M-matrix (the off-diagonal elements of $A$ are nonnegative) and $\Omega$ is the region where the model makes biological sense. If System (2) satisfies the above two conditions then the following theorem holds:

Theorem 3.2 The fixed point $U_{0}=\left(X^{*}, 0\right)$ is a globally asymptotic stable (GAS) equilibrium of (2) provided that $R_{0}<1$ (LAS) and that assumptions $(H 1)$ and $(H 2)$ are satisfied

$$
\begin{aligned}
& \frac{d X}{d t}=F(X, I)=\left(\begin{array}{c}
\pi_{\mathrm{H}}-\frac{\beta_{\mathrm{M}} b\left(I_{\mathrm{VS}}+\eta_{\mathrm{R}} I_{\mathrm{VR}}\right)}{N_{\mathrm{H}}} S_{\mathrm{H}}-\frac{B_{\mathrm{C}} \varphi}{\kappa+B_{\mathrm{C}}} S_{\mathrm{H}}-\mu_{\mathrm{H}} S_{\mathrm{H}}+\omega_{\mathrm{M}} R_{\mathrm{M}}+\omega_{\mathrm{C}} R_{\mathrm{C}}+\omega_{\mathrm{MC}} R_{\mathrm{MC}} \\
\alpha_{\mathrm{MS}} I_{\mathrm{US}}^{\mathrm{M}}+\alpha_{\mathrm{MT}} I_{\mathrm{TS}}^{\mathrm{M}}+\alpha_{\mathrm{MR}} I_{\mathrm{R}}^{\mathrm{M}}-\left(\mu+\omega_{\mathrm{M}}\right) R_{\mathrm{M}}-\frac{B_{\mathrm{C}} \alpha}{\kappa+B_{\mathrm{C}}} R_{\mathrm{M}} \\
\gamma_{\mathrm{C}} I_{\mathrm{C}}-\left(\mu_{\mathrm{H}}+\omega_{\mathrm{C}}\right) R_{\mathrm{C}}-\frac{\beta_{\mathrm{M}} b\left(I_{\mathrm{VS}}+\eta_{\mathrm{R}} I_{\mathrm{VR}}\right)}{N_{\mathrm{H}}} R_{\mathrm{C}} \\
\theta_{1}(1-f)(1-h) I_{\mathrm{USC}}^{\mathrm{M}}+\theta_{2}(1-f)(1-h) I_{\mathrm{TSC}}^{\mathrm{M}}+\theta_{3}(1-f)(1-h) I_{\mathrm{RC}}^{\mathrm{M}}-\left(\omega_{\mathrm{MC}}+\mu_{\mathrm{H}}\right) R_{\mathrm{MC}} \\
\pi_{\mathrm{v}}-\frac{\beta_{\mathrm{v}} b\left(I_{\mathrm{US}}^{\mathrm{M}}+\eta_{\mathrm{I}} I_{\mathrm{TS}}^{\mathrm{M}}+\eta_{\mathrm{R}} I_{\mathrm{R}}^{\mathrm{M}}\right)}{N_{\mathrm{H}}} S_{\mathrm{V}}-\frac{\beta_{\mathrm{v}} b\left(I_{\mathrm{USC}}^{\mathrm{M}}+\eta_{\mathrm{T}} I_{\mathrm{TSC}}^{\mathrm{M}}+\eta_{\mathrm{R}} I_{\mathrm{RC}}^{\mathrm{M}}\right)}{N_{\mathrm{H}}} S_{\mathrm{v}}-\mu_{\mathrm{v}} S_{\mathrm{V}}
\end{array}\right)
\end{aligned}
$$

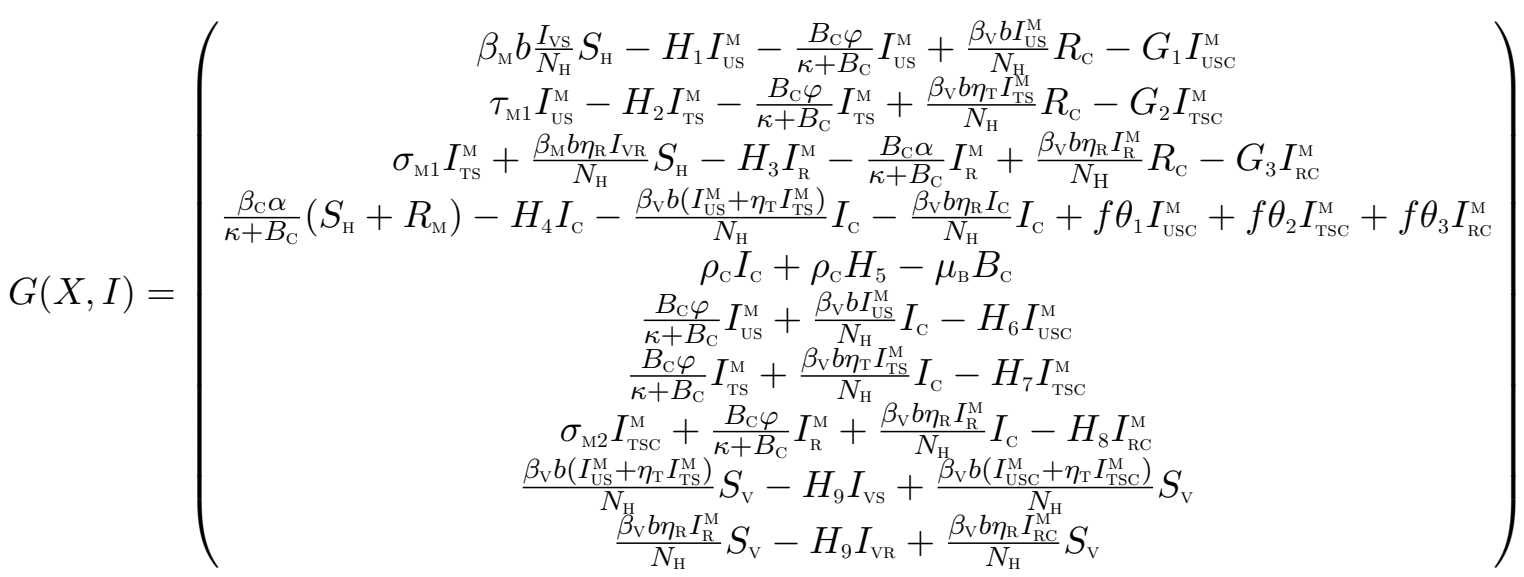

where $X$ denotes the number of non-infectious individuals and $I$ denotes the number of infected individuals.

$$
F(X, 0)=\left(\begin{array}{c}
\pi_{\mathrm{H}}-\mu_{\mathrm{H}} S_{\mathrm{H}} \\
0 \\
0 \\
0 \\
\pi_{\mathrm{v}}-\mu S_{\mathrm{v}}
\end{array}\right)
$$


medRxiv preprint doi: https://doi.org/10.1101/2020.08.18.20177329; this version posted September 10, 2020. The copyright holder for this preprint (which was not certified by peer review) is the author/funder, who has granted medRxiv a license to display the preprint in

It is made available under a CC-BY-NC-ND 4.0 International license .

$$
\begin{aligned}
& A=\left(\begin{array}{cccccccccc}
-H_{1} & 0 & 0 & 0 & 0 & G_{1} & 0 & 0 & \beta_{\mathrm{M}} b & 0 \\
\tau_{\mathrm{M} 1} & -H_{2} & 0 & 0 & 0 & 0 & G_{2} & 0 & 0 & 0 \\
0 & \sigma_{\mathrm{M} 1} & -H_{3} & 0 & 0 & 0 & 0 & G_{3} & 0 & \beta_{\mathrm{M}} \eta_{\mathrm{R}} b \\
0 & 0 & 0 & -H_{4} & \frac{\varphi S_{\mathrm{H}}^{*}}{\kappa} & f \theta_{1} & f \theta_{2} & f \theta_{3} & 0 & 0 \\
0 & 0 & 0 & \rho_{\mathrm{C}} & -\mu_{\mathrm{B}} & \rho_{\mathrm{C}} & \rho_{\mathrm{C}} \sigma_{\mathrm{T}} & \rho_{\mathrm{C}} \sigma_{\mathrm{R}} & 0 & 0 \\
0 & 0 & 0 & 0 & 0 & -H_{5} & 0 & 0 & 0 & 0 \\
0 & 0 & 0 & 0 & 0 & \tau_{\mathrm{M} 2} & -H_{6} & 0 & 0 & 0 \\
0 & 0 & 0 & 0 & 0 & 0 & \sigma_{\mathrm{M} 2} & -H_{7} & 0 & 0 \\
\frac{\beta_{\mathrm{V}} b S_{\mathrm{H}}^{*}}{N_{\mathrm{H}}^{*}} & \frac{\beta_{\mathrm{V}} b \eta_{\mathrm{T}} S_{\mathrm{H}}^{*}}{N_{\mathrm{H}}^{*}} & 0 & 0 & 0 & \frac{\beta_{\mathrm{V}} b S_{\mathrm{H}}^{*}}{N_{\mathrm{H}}^{*}} & \frac{\beta_{\mathrm{V}} b \eta_{\mathrm{T}} S_{\mathrm{H}}^{*}}{N_{\mathrm{H}}^{*}} & 0 & -\mu_{\mathrm{V}} & 0 \\
0 & 0 & \frac{\beta_{\mathrm{V}} b \eta_{\mathrm{R}} S_{\mathrm{H}}^{*}}{N_{\mathrm{H}}^{*}} & 0 & 0 & 0 & 0 & \frac{\beta_{\mathrm{V}} b \eta_{\mathrm{R}} S_{\mathrm{H}}^{*}}{N_{\mathrm{H}}^{*}} & 0 & -\mu_{\mathrm{V}}
\end{array}\right)
\end{aligned}
$$

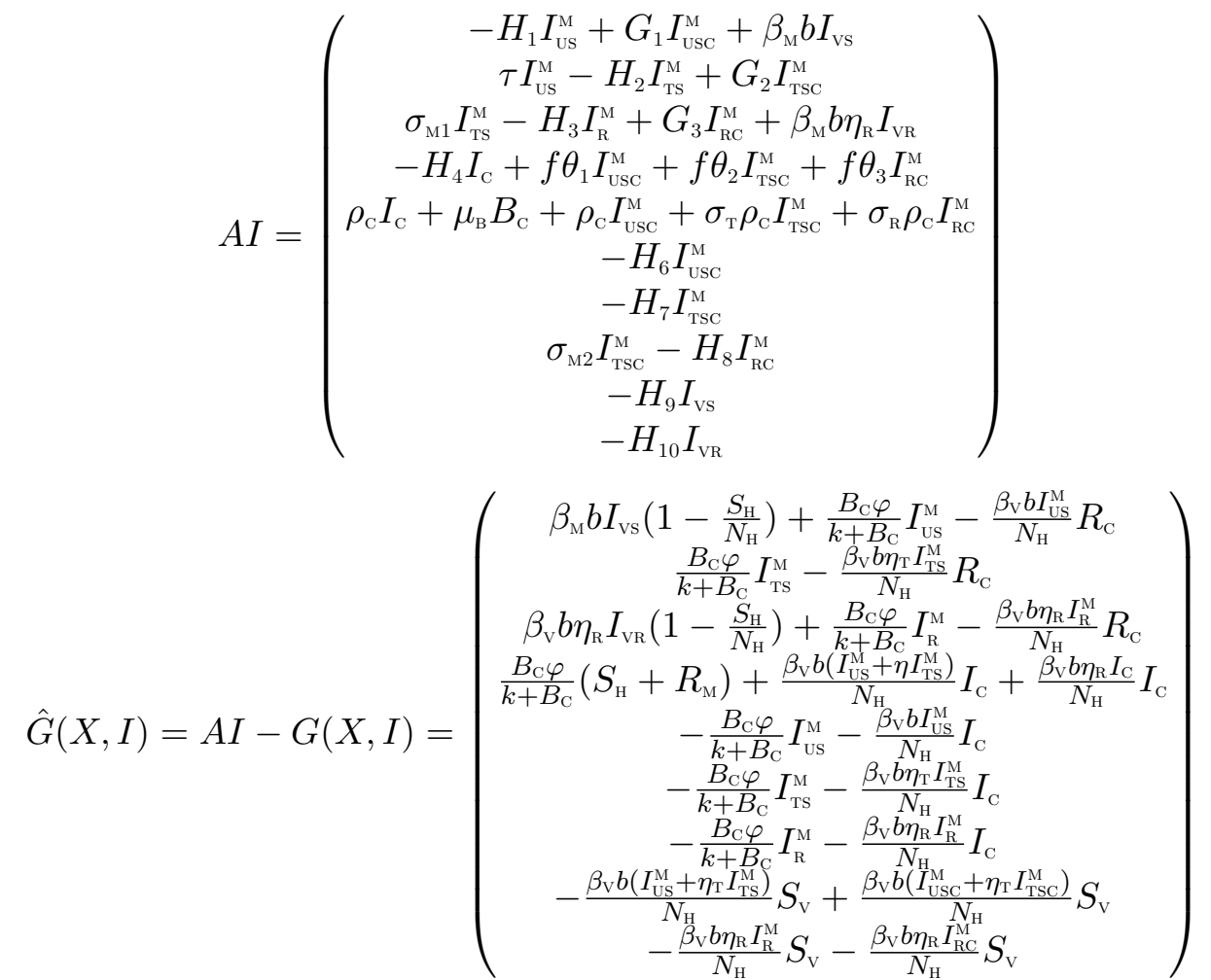

It is clear from the above, that, $\hat{G}(X, I) \nsupseteq 0$. Hence the DFE may not be globally asymptotically stable, suggesting the possibility of a backward bifurcation.

\subsection{Backward bifurcation analysis of the full co-infection model (2)}

In this section, we shall seek to determine the type of bifurcation the model (2) will exhibit, using the approach illustrated by Castillo-Chavez and Song [23. We establish the result below

Theorem 3.3 Suppose a backward bifurcation coefficient $a>0$, (with a defined below), when $\mathcal{R}_{0}<1$

$$
\begin{aligned}
a & =-\frac{2 \beta_{\mathrm{M}} b\left(\nu_{2} \omega_{14}+\eta_{\mathrm{R}} \nu_{4} \omega_{15}\right)}{N_{\mathrm{H}}^{*}}\left(\omega_{2}+\omega_{3}+\omega_{4}+\omega_{5}+\omega_{6}\right)-\frac{2 \beta_{\mathrm{M}} b \omega_{6}}{N_{\mathrm{H}}^{*}}\left[\left(\omega_{14}+\eta_{\mathrm{R}} \omega_{15}\right) \nu_{6}-\nu_{9} \omega_{14}-\nu_{11} \omega_{15}\right] \\
& -\frac{2 \beta_{\mathrm{V}} b \nu_{14}\left(\omega_{2}+\eta_{\mathrm{T}} \omega_{3}\right)}{N_{\mathrm{H}}^{* 2}}\left[\left(\omega_{1}+\omega_{2}+\omega_{3}+\omega_{4}+\omega_{5}+\omega_{6}+\omega_{7}\right) x_{13}^{*}-\omega_{13} N_{\mathrm{H}}^{*}\right] \\
& -\frac{2 \beta_{\mathrm{v}} \eta_{\mathrm{R}} b \nu_{15} \omega_{4}}{N_{\mathrm{H}}^{* 2}}\left[\left(\omega_{1}+\omega_{2}+\omega_{3}+\omega_{4}+\omega_{5}+\omega_{6}+\omega_{7}\right) x_{13}^{*}-\omega_{13} N_{\mathrm{H}}^{*}\right]
\end{aligned}
$$

then model (2) undergoes the phenomenon of backward bifurcation at $\mathcal{R}_{0}=1$. If a $<0$, then the system (2) exhibits a forward bifurcation at $\mathcal{R}_{0}=1$.

\section{Proof}

Suppose

$$
\xi_{e}=\left(S_{\mathrm{H}}^{* *}, I_{\mathrm{us}}^{\mathrm{M}^{* *}}, I_{\mathrm{Ts}}^{\mathrm{M}^{* *}}, I_{\mathrm{R}}^{\mathrm{M}^{* *}}, R_{\mathrm{M}}^{* *}, I_{\mathrm{c}}^{* *}, R_{\mathrm{c}}^{* *}, B_{\mathrm{c}}^{* *}, I_{\mathrm{usc}}^{\mathrm{M}^{* *}}, I_{\mathrm{TsC}}^{\mathrm{M}^{* *}}, I_{\mathrm{RC}}^{\mathrm{M}^{* *}}, R_{\mathrm{MC}}^{* *}, S_{\mathrm{v}}^{* *}, I_{\mathrm{vs}}^{* *}, I_{\mathrm{vR}}^{* *}\right)
$$


medRxiv preprint doi: https://doi.org/10.1101/2020.08.18.20177329; this version posted September $10,2020$. The copyright holder for this preprint (which was not certified by peer review) is the author/funder, who has granted medRxiv a license to display the preprint in

It is made available under a CC-BY-NC-ND 4.0 International license .

represents any arbitrary endemic equilibrium of the model. The existence of backward bifurcation will be studied using the Centre Manifold Theory by Castillo-Chavez and Song [23. To apply this theory, it is appropriate to do the following change of variables.

Let

$$
\begin{aligned}
S_{\mathrm{H}} & =x_{1}, I_{\mathrm{US}}^{\mathrm{M}}=x_{2}, I_{\mathrm{TS}}^{\mathrm{M}}=x_{3}, I_{\mathrm{R}}^{\mathrm{M}}=x_{4}, R_{\mathrm{M}}=x_{5}, I_{\mathrm{C}}=x_{6}, R_{\mathrm{C}}=x_{7}, B_{\mathrm{C}}=x_{8}, I_{\mathrm{USC}}^{\mathrm{M}}=x_{9}, I_{\mathrm{TSC}}^{\mathrm{M}}=x_{10}, I_{\mathrm{rC}}^{\mathrm{M}}=x_{11}, \\
R_{\mathrm{MC}} & =x_{12}, S_{\mathrm{v}}=x_{13}, I_{\mathrm{vS}}=x_{14}, I_{\mathrm{vR}}=x_{15}
\end{aligned}
$$

Moreover, using the vector notation

$$
X=\left(x_{1}, x_{2}, x_{3}, x_{4}, x_{5}, x_{6}, x_{7}, x_{8}, x_{9}, x_{10}, x_{11}, x_{12}, x_{13}, x_{14}, x_{15}\right)^{T}
$$

the model (2) can be re-written in the form

$$
\frac{d X}{d t}=f=\left(f_{1}, f_{2}, f_{3}, f_{4}, f_{5}, f_{6}, f_{7}, f_{8}, f_{9}, f_{10}, f_{11}, f_{12}, f_{13}, f_{14}, f_{15}\right)^{T}
$$

as follows:

$$
\begin{aligned}
& \frac{d x_{1}}{d t}=\pi_{\mathrm{H}}-\frac{\beta_{\mathrm{M}} b\left(x_{14}+\eta_{\mathrm{R}} x_{15}\right)}{N_{\mathrm{H}}} x_{1}-\frac{x_{8} \varphi}{\kappa+x_{8}} x_{1}-\mu_{\mathrm{H}} x_{1}+\omega_{\mathrm{M}} x_{5}+\omega_{\mathrm{C}} x_{7}+\omega_{\mathrm{MC}} x_{12}:=f_{1} \\
& \frac{d x_{2}}{d t}=\beta_{\mathrm{M}} b \frac{x_{14}}{N_{\mathrm{H}}} x_{1}-\left(\tau_{\mathrm{M} 1}+\mu_{\mathrm{H}}+\alpha_{\mathrm{MS}}+\delta_{\mathrm{MS} 1}\right) x_{2}-\frac{x_{8} \varphi}{\kappa+x_{8}} x_{2}+\frac{\beta_{\mathrm{M}} b x_{14}}{N_{\mathrm{H}}} x_{7}+h \theta_{1}(1-f) x_{9}:=f_{2} \\
& \frac{d x_{3}}{d t}=\tau_{\mathrm{M} 1} x_{2}-\left(\sigma_{\mathrm{M} 1}+\mu_{\mathrm{H}}+\alpha_{\mathrm{MT}}+\phi_{1} \delta_{\mathrm{MS} 1}\right) x_{3}-\frac{x_{8} \varphi}{\kappa+x_{8}} x_{3}+h \theta_{2}(1-f) x_{10}:=f_{3} \\
& \frac{d x_{4}}{d t}=\sigma_{\mathrm{M} 1} x_{3}+\frac{\beta_{\mathrm{M}} b \eta_{\mathrm{R}} x_{15}}{N_{\mathrm{H}}} x_{1}-\left(\mu_{\mathrm{H}}+\alpha_{\mathrm{MR}}+\delta_{\mathrm{MR} 1}\right) x_{4}-\frac{x_{8} \varphi}{\kappa+x_{8}} x_{4}+\frac{\beta_{\mathrm{M}} b \eta_{\mathrm{R}} x_{15}}{N_{\mathrm{H}}} x_{7}+h \theta_{3}(1-f) x_{11}:=f_{4} \\
& \frac{d x_{5}}{d t}=\alpha_{\mathrm{MS}} x_{2}+\alpha_{\mathrm{MT}} x_{3}+\alpha_{\mathrm{MR}} x_{4}-\left(\mu+\omega_{\mathrm{M}}\right) x_{5}-\frac{x_{8} \varphi}{\kappa+x_{8}} x_{5}:=f_{5} \\
& \frac{d x_{6}}{d t}=\frac{x_{8} \varphi}{\kappa+x_{8}}\left(x_{1}+x_{5}\right)-\left(\delta_{\mathrm{c} 1}+\mu_{\mathrm{H}}+\gamma_{\mathrm{c}}\right) x_{6}-\frac{\beta_{\mathrm{M}} b x_{14}}{N_{\mathrm{H}}} x_{6}-\frac{\beta_{\mathrm{M}} b \eta_{\mathrm{R}} x_{15}}{N_{\mathrm{H}}} x_{6} \\
& +f \theta_{1} x_{9}+f \theta_{2} x_{10}+f \theta_{3} x_{11}:=f_{6} \\
& \frac{d x_{7}}{d t}=\gamma_{\mathrm{C}} x_{6}-\left(\mu_{\mathrm{H}}+\omega_{\mathrm{c}}\right) x_{7}-\frac{\beta_{\mathrm{M}} b\left(x_{14}+\eta_{\mathrm{R}} x_{15}\right)}{N_{\mathrm{H}}} x_{7}:=f_{7} \\
& \frac{d x_{8}}{d t}=\rho_{\mathrm{C}} x_{6}+\rho_{\mathrm{C}}\left(x_{9}+\sigma_{\mathrm{T}} x_{10}+\sigma_{\mathrm{R}} x_{11}\right)-\mu_{\mathrm{B}} x_{8}:=f_{8} \\
& \frac{d x_{9}}{d t}=\frac{x_{8} \varphi}{\kappa+x_{8}} x_{2}+\frac{\beta_{\mathrm{M}} b x_{14}}{N_{\mathrm{H}}} x_{6}-\left(\mu_{\mathrm{H}}+\delta_{\mathrm{MS} 2}+\delta_{\mathrm{C} 2}+\tau_{\mathrm{M} 2}+\theta_{1}\right) x_{9}:=f_{9} \\
& \frac{d x_{10}}{d t}=\frac{x_{8} \varphi}{\kappa+x_{8}} x_{3}+\tau_{\mathrm{M} 2} x_{9}-\left(\mu_{\mathrm{H}}+\phi_{2} \delta_{\mathrm{MS} 2}+\theta_{2}+\sigma_{\mathrm{M} 2}+\delta_{\mathrm{C} 2}\right) x_{10}:=f_{10} \\
& \frac{d x_{11}}{d t}=\sigma_{\mathrm{M} 2} x_{10}+\frac{x_{8} \varphi}{\kappa+x_{8}} x_{4}+\frac{\beta_{\mathrm{M}} b \eta_{\mathrm{R}} x_{15}}{N_{\mathrm{H}}} x_{6}-\left(\mu_{\mathrm{H}}+\delta_{\mathrm{MR} 2}+\delta_{\mathrm{C} 2}+\theta_{3}\right) x_{11}:=f_{11} \\
& \frac{d x_{12}}{d t}=\theta_{1}(1-f)(1-h) x_{9}+\theta_{2}(1-f)(1-h) x_{10}+\theta_{3}(1-f)(1-h) x_{11}-\left(\omega_{\mathrm{MC}}+\mu_{\mathrm{H}}\right) x_{12}:=f_{12} \\
& \frac{d x_{13}}{d t}=\pi_{\mathrm{v}}-\frac{\beta_{\mathrm{v}} b\left(x_{2}+\eta_{\mathrm{T}} x_{3}+\eta_{\mathrm{R}} x_{4}\right)}{N_{\mathrm{H}}} x_{13}-\frac{\beta_{\mathrm{v}} b\left(x_{9}+\eta_{\mathrm{T}} x_{10}+\eta_{\mathrm{R}} x_{11}\right)}{N_{\mathrm{H}}} x_{13}-\mu_{\mathrm{v}} x_{13}:=f_{13} \\
& \frac{d x_{14}}{d t}=\frac{\beta_{\mathrm{v}} b\left(x_{2}+\eta_{\mathrm{T}} x_{3}\right)}{N_{\mathrm{H}}} x_{13}+\frac{\beta_{\mathrm{v}} b\left(x_{9}+\eta_{\mathrm{T}} x_{10}\right)}{N_{\mathrm{H}}} x_{13}-\mu_{\mathrm{v}} x_{14}:=f_{14} \\
& \frac{d x_{15}}{d t}=\frac{\beta_{\mathrm{v}} b \eta_{\mathrm{R}} x_{4}}{N_{\mathrm{H}}} x_{13}+\frac{\beta_{\mathrm{v}} b \eta_{\mathrm{R}} x_{11}}{N_{\mathrm{H}}} x_{13}-\mu_{\mathrm{v}} x_{15}:=f_{15}
\end{aligned}
$$


medRxiv preprint doi: https://doi.org/10.1101/2020.08.18.20177329; this version posted September $10,2020$. The copyright holder for this preprint (which was not certified by peer review) is the author/funder, who has granted medRxiv a license to display the preprint in

It is made available under a CC-BY-NC-ND 4.0 International license .

$J\left(\xi_{0}\right)=\left(\begin{array}{ccccccccccccccc}-\mu_{\mathrm{H}} & 0 & 0 & 0 & \omega_{\mathrm{M}} & 0 & \omega_{\mathrm{C}} & -\frac{\varphi x_{1}^{*}}{\kappa} & 0 & 0 & 0 & \omega_{\mathrm{MC}} & 0 & -\beta_{\mathrm{M}} b & -\beta_{\mathrm{M}} \eta_{\mathrm{R}} b \\ 0 & -H_{1} & 0 & 0 & 0 & 0 & 0 & 0 & G_{1} & 0 & 0 & 0 & 0 & \beta_{\mathrm{M}} b & 0 \\ 0 & \tau_{\mathrm{M} 1} & -H_{2} & 0 & 0 & 0 & 0 & 0 & 0 & G_{2} & 0 & 0 & 0 & 0 & 0 \\ 0 & 0 & \sigma_{\mathrm{M} 1} & -H_{3} & 0 & 0 & 0 & 0 & 0 & 0 & G_{3} & 0 & 0 & 0 & 0 \\ \alpha_{\mathrm{M} 2} & \alpha_{\mathrm{MT}} & \alpha_{\mathrm{MR}} & -D_{1} & 0 & 0 & 0 & 0 & 0 & 0 & 0 & 0 & 0 & 0 & 0 \\ 0 & 0 & 0 & 0 & 0 & -H_{4} & 0 & \frac{\varphi x_{1}^{*}}{\kappa} & f \theta_{1} & f \theta_{2} & f \theta_{3} & 0 & 0 & 0 & 0 \\ 0 & 0 & 0 & 0 & 0 & \gamma_{\mathrm{C}} & -D_{2} & 0 & 0 & 0 & 0 & 0 & 0 & 0 & 0 \\ 0 & 0 & 0 & 0 & 0 & \rho_{\mathrm{C}} & 0 & -\mu_{\mathrm{B}} & \rho_{\mathrm{C}} & \rho_{\mathrm{C}} \sigma_{\mathrm{T}} & \rho_{\mathrm{C}} \sigma_{\mathrm{R}} & 0 & 0 & 0 & 0 \\ 0 & 0 & 0 & 0 & 0 & 0 & 0 & 0 & -H_{5} & 0 & 0 & 0 & 0 & 0 & 0 \\ 0 & 0 & 0 & 0 & 0 & 0 & 0 & 0 & \tau_{\mathrm{M} 2} & -H_{6} & 0 & 0 & 0 & 0 & 0 \\ 0 & 0 & 0 & 0 & 0 & 0 & 0 & 0 & 0 & \sigma_{\mathrm{M} 2} & -H_{7} & 0 & 0 & 0 & 0 \\ 0 & 0 & 0 & 0 & 0 & 0 & 0 & 0 & G_{4} & G_{5} & G_{6} & -D D_{3} & 0 & 0 & 0 \\ 0 & -\Phi & -\Phi \eta_{\mathrm{T}} & -\Phi \eta_{\mathrm{R}} & 0 & 0 & 0 & 0 & -\Phi & -\Phi \eta_{\mathrm{T}} & -\Phi \eta_{\mathrm{R}} & 0 & -\mu_{\mathrm{V}} & 0 & 0 \\ 0 & \Phi & \Phi \eta_{\mathrm{T}} & 0 & 0 & 0 & 0 & 0 & \Phi & \Phi \eta_{\mathrm{T}} & 0 & 0 & 0 & -\mu_{\mathrm{V}} & 0 \\ 0 & 0 & 0 & \Phi \eta_{\mathrm{R}} & 0 & 0 & 0 & 0 & 0 & 0 & \Phi \eta_{\mathrm{R}} & 0 & 0 & 0 & -\mu_{\mathrm{V}}\end{array}\right)$

with,

$H_{1}=\tau_{\mathrm{M} 1}+\mu_{\mathrm{H}}+\alpha_{\mathrm{MS}}+\delta_{\mathrm{MS} 1}, \quad H_{2}=\sigma_{\mathrm{M} 1}+\mu_{\mathrm{H}}+\alpha_{\mathrm{MT}}+\phi_{1} \delta_{\mathrm{MS} 1}, \quad H_{3}=\mu_{\mathrm{H}}+\alpha_{\mathrm{MR}}+\delta_{\mathrm{MR} 1}, \quad H_{4}=\delta_{\mathrm{C} 1}+\mu_{\mathrm{H}}+\gamma_{\mathrm{C}}$

$H_{5}=\mu_{\mathrm{H}}+\delta_{\mathrm{MS} 2}+\delta_{\mathrm{C} 2}+\tau_{\mathrm{M} 2}+\theta_{1}, \quad H_{6}=\phi_{1} \delta_{\mathrm{MS} 2}+\mu_{\mathrm{H}}+\theta_{2} \sigma_{\mathrm{M} 2}+\delta_{\mathrm{C} 2}, \quad H_{7}=\delta_{\mathrm{MR} 2}+\mu_{\mathrm{H}}+\delta_{\mathrm{C} 2}+\theta_{3}$

$G_{1}=h \theta_{1}(1-f), \quad G_{2}=h \theta_{2}(1-f), \quad G_{3}=h \theta_{3}(1-f), \quad G_{4}=\theta_{1}(1-f)(1-h), \quad G_{5}=\theta_{2}(1-f)(1-h)$

$G_{6}=\theta_{3}(1-f)(1-h), \quad D_{1}=\mu_{\mathrm{H}}+\omega_{\mathrm{M}}, \quad D_{2}=\mu_{\mathrm{H}}+\omega_{\mathrm{C}}, \quad D_{3}=\mu_{\mathrm{H}}+\omega_{\mathrm{MC}}, \quad \Phi=\frac{\beta_{\mathrm{v}} b x_{13}^{*}}{N_{\mathrm{H}}^{*}}$

Consider the case when $\mathcal{R}_{0 \mathrm{MR}}=1$. Assume, further, that thee product $\beta_{\mathrm{M}} \eta_{\mathrm{R}}$ is chosen as a bifurcation parameter. Solving for $\beta_{\mathrm{M}} \eta_{\mathrm{R}}=\beta^{*}$ from $\mathcal{R}_{0 \mathrm{MR}}=1$ gives

$$
\beta_{\mathrm{M}}^{*} \eta_{\mathrm{R}}^{*}=\beta^{*}=\frac{\mu_{\mathrm{v}} N_{\mathrm{H}}^{*} H_{3}}{b \eta_{\mathrm{R}} \beta_{\mathrm{v}} S_{\mathrm{V}}^{*}}
$$

Evaluating the Jacobian of the system (9) at the DFE, $J\left(\xi_{0}\right)$, and using the approach in Castillo-Chavez and Song [23, we have that $J\left(\xi_{0}\right)$ has a right eigenvector (associated with the non-zero eigenvalue) given by

$$
\mathbf{w}=\left[\omega_{1}, \omega_{2}, \omega_{3}, \omega_{4}, \omega_{5}, \omega_{6}, \omega_{7}, \omega_{8}, \omega_{9}, \omega_{10}, \omega_{11}, \omega_{12}, \omega_{13}, \omega_{14}, \omega_{15}\right]^{T}
$$

where,

$$
\begin{aligned}
\omega_{1} & =\frac{1}{\mu_{\mathrm{H}}}\left[\omega_{\mathrm{M}} \omega_{5}+\frac{\omega_{\mathrm{c}} \gamma_{\mathrm{C}} \varphi x_{1}^{*}}{\mu_{\mathrm{B}} \kappa D_{2} H_{4}}-\frac{H_{1}}{\Phi\left(1+\eta_{\mathrm{T}} \tau_{\mathrm{M} 1}\right)}-\frac{\beta_{\mathrm{v}} \beta_{\mathrm{M}}^{2} \eta_{\mathrm{R}}^{2} b_{3} \sigma_{\mathrm{M} 1} \tau_{\mathrm{M} 1}}{\mu_{\mathrm{v}}^{3} N_{\mathrm{H}}^{*}(1-b) H_{1} H_{3}}\right] \\
\omega_{2} & =\frac{\beta_{\mathrm{M}} b}{\mu_{\mathrm{v}} H_{1}}, \quad \omega_{3}=\frac{\beta_{\mathrm{M}} b \tau_{\mathrm{M} 1}}{\mu_{\mathrm{v}} H_{1}}, \quad \omega_{4}=\frac{\sigma_{\mathrm{M} 1} \beta_{\mathrm{M}} b \tau_{\mathrm{M} 1}}{\mu_{\mathrm{v}}^{2}(1-b) H_{1} H_{3}} \\
\omega_{5} & =\frac{\beta_{\mathrm{M}} b\left[\alpha_{\mathrm{M} 2} \mu_{\mathrm{v}}(1-b) H_{3}+\alpha_{\mathrm{MT}} \mu_{\mathrm{v}}(1-b) H_{3} \tau_{\mathrm{M} 1}+\alpha_{\mathrm{MR}} \sigma_{\mathrm{M} 1} \beta_{\mathrm{M}} b \tau_{\mathrm{M} 1}\right]}{\mu_{\mathrm{v}}^{2}(1-b) H_{1} H_{3} D_{1}} \\
\omega_{6} & =\frac{\varphi x_{1}^{*}}{\mu_{\mathrm{B}} \kappa H_{4}}, \quad \omega_{7}=\frac{\gamma_{\mathrm{c}} \varphi x_{1}^{*}}{\mu_{\mathrm{B}} \kappa H_{4} D_{2}}, \quad \omega_{8}=\frac{H_{4} \kappa}{\rho_{\mathrm{C}} \varphi x_{1}^{*}} \\
\omega_{9} & =\omega_{10}=\omega_{11}=\omega_{12}=0 \\
\omega_{13} & =-\frac{1}{\mu_{\mathrm{v}}}\left[\frac{\Phi \beta_{\mathrm{M}} b\left(1+\eta_{\mathrm{T}} \tau_{\mathrm{M} 1}\right)}{\mu_{\mathrm{v}} H_{1}}+\frac{\Phi \eta_{\mathrm{R}} \sigma_{\mathrm{M} 1} \beta_{\mathrm{M}} b \tau_{\mathrm{M} 1}}{\mu_{\mathrm{v}}^{2}(1-b) H_{1} H_{3}}\right], \quad \omega_{14}=\frac{H_{1}}{\beta_{\mathrm{M}} b \Phi\left(1+\eta_{\mathrm{T}} \tau_{\mathrm{M} 1}\right)} \\
\omega_{15} & =\frac{\beta_{\mathrm{v}} \beta_{\mathrm{M}} \eta_{\mathrm{R}} \sigma_{\mathrm{M} 1} \tau_{\mathrm{M} 1} b^{2}}{\mu_{\mathrm{v}}^{3} N_{\mathrm{H}}^{*}(1-b) H_{1} H_{3}}
\end{aligned}
$$


medRxiv preprint doi: https://doi.org/10.1101/2020.08.18.20177329; this version posted September 10, 2020. The copyright holder for this preprint (which was not certified by peer review) is the author/funder, who has granted medRxiv a license to display the preprint in

Similarly, the components of the left eigenvector of $\left.J\left(\xi_{0}\right)\right|_{\beta=\beta_{\mathrm{M}}^{*} \eta_{\mathrm{R}}^{*}}, \mathbf{v}=\left(\nu_{1}, \nu_{2}, \ldots, \nu_{15}\right)$, satisfying $\mathbf{v} \cdot \mathbf{w}=1$, are given by:

$$
\begin{aligned}
& \nu_{1}=\nu_{5}=\nu_{7}=\nu_{12}=\nu_{13}=0, \quad \nu_{2}=\nu_{2}>0 \\
& \nu_{3}=\frac{1}{H_{2}}\left[\frac{\sigma_{\mathrm{M} 1} \Phi \eta_{\mathrm{R}}}{\mu_{\mathrm{v}} H_{3}}+\frac{\Phi \eta_{\mathrm{T}} \beta_{\mathrm{M}} b \nu_{2}}{\mu_{\mathrm{v}}}\right], \quad \nu_{4}=\frac{\Phi \eta_{\mathrm{R}}}{\mu_{\mathrm{v}} H_{3}}, \quad \nu_{6}=\frac{\rho_{\mathrm{C}}}{\mu_{\mathrm{B}} H_{1}}, \quad \nu_{8}=\frac{\kappa H_{1}}{\rho_{\mathrm{C}} \varphi x_{1}^{*}} \\
& \nu_{9}=\frac{\mu_{\mathrm{B}} H_{1} G_{2} \varphi x_{1}^{*} \nu_{2}+\varphi x_{1}^{*} f \theta_{1} \rho_{\mathrm{C}}+\mu_{\mathrm{B}} H_{1}^{2} \kappa}{\mu_{\mathrm{B}} H_{1} H_{5} \varphi x_{1}^{*}}, \quad \nu_{10}=\frac{G_{2} \nu_{3}+f \theta_{2} \nu_{6}+\rho_{\mathrm{C}} \sigma_{\mathrm{T}} \nu_{8}+\sigma_{\mathrm{M} 2} \nu_{11}}{H_{6}} \\
& \nu_{11}=\frac{G_{3} \nu_{4}+f \theta_{3} \nu_{6}+\rho_{\mathrm{C}} \sigma_{\mathrm{R}} \nu_{8}+\Phi \eta_{\mathrm{R}} \nu_{15}}{H_{7}}, \quad \nu_{14}=\frac{\beta_{\mathrm{M}} b \nu_{2}}{\mu_{\mathrm{v}}}, \quad \nu_{15}=\frac{H_{3}}{\Phi \beta_{\mathrm{M}} b \eta_{\mathrm{R}}^{2}}
\end{aligned}
$$

The non-zero second partial derivatives of the functions $f_{i}(i=1, \ldots, 15)$ are given by

$$
\begin{aligned}
& \frac{\partial^{2} f_{2}}{\partial x_{1} \partial x_{14}}=\frac{\partial^{2} f_{2}}{\partial x_{7} \partial x_{14}}=-\frac{\beta_{\mathrm{M}} b x_{1}^{*}}{N_{\mathrm{H}}^{* 2}}+\frac{\beta_{\mathrm{M}} b}{N_{\mathrm{H}}^{*}}, \\
& \frac{\partial^{2} f_{2}}{\partial x_{2} \partial x_{14}}=\frac{\partial^{2} f_{2}}{\partial x_{3} \partial x_{14}}=\frac{\partial^{2} f_{2}}{\partial x_{4} \partial x_{14}}=\frac{\partial^{2} f_{2}}{\partial x_{5} \partial x_{14}}=\frac{\partial^{2} f_{2}}{\partial x_{6} \partial x_{14}}=-\frac{\beta_{\mathrm{M}} b x_{1}^{*}}{N_{\mathrm{H}}^{* 2}} \\
& \frac{\partial^{2} f_{4}}{\partial x_{1} \partial x_{15}}=\frac{\partial^{2} f_{4}}{\partial x_{7} \partial x_{15}}=-\frac{\beta_{\mathrm{M}} b \eta_{\mathrm{R}} x_{1}^{*}}{N_{\mathrm{H}}^{* 2}}+\frac{\beta_{\mathrm{M}} b \eta_{\mathrm{R}}}{N_{\mathrm{H}}^{*}}, \\
& \frac{\partial^{2} f_{4}}{\partial x_{2} \partial x_{15}}=\frac{\partial^{2} f_{4}}{\partial x_{3} \partial x_{15}}=\frac{\partial^{2} f_{4}}{\partial x_{4} \partial x_{15}}=\frac{\partial^{2} f_{4}}{\partial x_{5} \partial x_{15}}=\frac{\partial^{2} f_{4}}{\partial x_{6} \partial x_{15}}=-\frac{\beta_{\mathrm{M}} b \eta_{\mathrm{R}} x_{1}^{*}}{N_{\mathrm{H}}^{* 2}} \\
& \frac{\partial^{2} f_{6}}{\partial x_{6} \partial x_{14}}=-\frac{\beta_{\mathrm{M}} b}{N_{\mathrm{H}}^{*}}, \quad \frac{\partial^{2} f_{6}}{\partial x_{6} \partial x_{15}}=-\frac{\beta_{\mathrm{M}} b \eta_{\mathrm{R}}}{N_{\mathrm{H}}^{*}}, \quad \frac{\partial^{2} f_{9}}{\partial x_{6} \partial x_{14}}=\frac{\beta_{\mathrm{M}} b}{N_{\mathrm{H}}^{*}}, \quad \frac{\partial^{2} f_{11}}{\partial x_{6} \partial x_{15}}=\frac{\beta_{\mathrm{M}} b \eta_{\mathrm{R}}}{N_{\mathrm{H}}^{*}} \\
& \frac{\partial^{2} f_{14}}{\partial x_{1} \partial x_{2}}=\frac{\partial^{2} f_{14}}{\partial x_{2} \partial x_{2}}=\frac{\partial^{2} f_{14}}{\partial x_{2} \partial x_{4}}=\frac{\partial^{2} f_{14}}{\partial x_{2} \partial x_{5}}=\frac{\partial^{2} f_{14}}{\partial x_{2} \partial x_{6}}=\frac{\partial^{2} f_{14}}{\partial x_{2} \partial x_{7}}=-\frac{\beta_{\mathrm{v}} b x_{13}^{*}}{N_{\mathrm{H}}^{*}} \\
& \frac{\partial^{2} f_{14}}{\partial x_{1} \partial x_{3}}=\frac{\partial^{2} f_{14}}{\partial x_{3} \partial x_{4}}=\frac{\partial^{2} f_{14}}{\partial x_{3} \partial x_{5}}=\frac{\partial^{2} f_{14}}{\partial x_{3} \partial x_{6}}=\frac{\partial^{2} f_{14}}{\partial x_{3} \partial x_{7}}=-\frac{\beta_{\mathrm{v}} b \eta_{\mathrm{T}} x_{13}^{*}}{N_{\mathrm{H}}^{*}} \\
& \frac{\partial^{2} f_{14}}{\partial x_{2} \partial x_{3}}=\frac{\beta_{\mathrm{v}} b x_{13}^{*}}{N_{\mathrm{H}}^{*}}-\frac{\beta_{\mathrm{v}} b x_{13}^{*} \eta_{\mathrm{T}}}{N_{\mathrm{H}}^{*}}, \quad \frac{\partial^{2} f_{14}}{\partial x_{3}^{2}}=-\frac{2 \beta_{\mathrm{v}} b x_{13}^{*} \eta_{\mathrm{T}}}{N_{\mathrm{H}}^{* 2}}, \quad \frac{\partial^{2} f_{14}}{\partial x_{2} \partial x_{13}}=\frac{\beta_{\mathrm{v}} b}{N_{\mathrm{H}}^{*}}, \quad \frac{\partial^{2} f_{14}}{\partial x_{3} \partial x_{3}}=\frac{\beta_{\mathrm{v}} b \eta_{\mathrm{T}}}{N_{\mathrm{H}}^{*}} \\
& \frac{\partial^{2} f_{15}}{\partial x_{1} \partial x_{4}}=\frac{\partial^{2} f_{15}}{\partial x_{2} \partial x_{4}}=\frac{\partial^{2} f_{15}}{\partial x_{3} \partial x_{4}}=\frac{\partial^{2} f_{15}}{\partial x_{4} \partial x_{5}}=\frac{\partial^{2} f_{15}}{\partial x_{4} \partial x_{6}}=\frac{\partial^{2} f_{15}}{\partial x_{4} \partial x_{7}}=-\frac{\beta_{\mathrm{v}} b \eta_{\mathrm{R}} x_{13}^{*}}{N_{\mathrm{H}}^{*}} \\
& \frac{\partial^{2} f_{15}}{\partial x_{4}^{2}}=-\frac{2 \beta_{\mathrm{v}} b \eta_{\mathrm{R}}}{N_{\mathrm{H}}^{* 2}}, \quad \frac{\partial^{2} f_{15}}{\partial x_{4} \partial x_{13}}=\frac{\beta_{\mathrm{v}} b \eta_{\mathrm{R}}}{N_{\mathrm{H}}^{*}}
\end{aligned}
$$

The associated bifurcation coefficients defined by $a$ and $b$, given by:

$$
a=\sum_{k, i, j=1}^{n} \nu_{k} \omega_{i} \omega_{j} \frac{\partial^{2} f_{k}}{\partial x_{i} \partial x_{j}}(0,0) \quad \text { and } \quad b=\sum_{k, i=1}^{n} \nu_{k} \omega_{i} \frac{\partial^{2} f_{k}}{\partial x_{i} \partial \beta_{\mathrm{HP}}^{*}}(0,0)
$$

are computed to be

$$
\begin{aligned}
a & =-\frac{2 \beta_{\mathrm{M}} b\left(\nu_{2} \omega_{14}+\eta_{\mathrm{R}} \nu_{4} \omega_{15}\right)}{N_{\mathrm{H}}^{*}}\left(\omega_{2}+\omega_{3}+\omega_{4}+\omega_{5}+\omega_{6}\right)-\frac{2 \beta_{\mathrm{M}} b \omega_{6}}{N_{\mathrm{H}}^{*}}\left[\left(\omega_{14}+\eta_{\mathrm{R}} \omega_{15}\right) \nu_{6}-\nu_{9} \omega_{14}-\nu_{11} \omega_{15}\right] \\
& -\frac{2 \beta_{\mathrm{v}} b \nu_{14}\left(\omega_{2}+\eta_{\mathrm{T}} \omega_{3}\right)}{N_{\mathrm{H}}^{* 2}}\left[\left(\omega_{1}+\omega_{2}+\omega_{3}+\omega_{4}+\omega_{5}+\omega_{6}+\omega_{7}\right) x_{13}^{*}-\omega_{13} N_{\mathrm{H}}^{*}\right] \\
& -\frac{2 \beta_{\mathrm{v}} \eta_{\mathrm{R}} b \nu_{15} \omega_{4}}{N_{\mathrm{H}}^{* 2}}\left[\left(\omega_{1}+\omega_{2}+\omega_{3}+\omega_{4}+\omega_{5}+\omega_{6}+\omega_{7}\right) x_{13}^{*}-\omega_{13} N_{\mathrm{H}}^{*}\right]
\end{aligned}
$$

and

$$
b=\sum_{k, i=1}^{20} \nu_{k} \omega_{i} \frac{\partial^{2} f_{k}}{\partial x_{i} \partial \beta^{*}}(0,0)=\left(\omega_{14}+\eta_{\mathrm{R}} \omega_{15}\right) \nu_{2}>0
$$

Since the bifurcation coefficient $b$ is positive, it follows from Theorem 4.1 in Castillo-Chavez and Song [23] that the model (2), or the transformed model (9), will undergo the phenomenon of backward bifurcation if the coefficient, $a$, given by 10 is positive. 


\section{Analysis of the optimal control model}

$$
\begin{aligned}
& \frac{d S_{\mathrm{H}}}{d t}=\pi_{\mathrm{H}}-\frac{\beta_{\mathrm{M}} b\left(I_{\mathrm{VS}}+\eta_{\mathrm{R}} I_{\mathrm{VR}}\right)}{N_{\mathrm{H}}} S_{\mathrm{H}}-\frac{B_{\mathrm{C}} \varphi}{\kappa+B_{\mathrm{C}}} S_{\mathrm{H}}-\mu_{\mathrm{H}} S_{\mathrm{H}}+\omega_{\mathrm{M}} R_{\mathrm{M}}+\omega_{\mathrm{C}} R_{\mathrm{C}}+\omega_{\mathrm{MC}} R_{\mathrm{MC}} \\
& \frac{d I_{\mathrm{US}}^{\mathrm{M}}}{d t}=\beta_{\mathrm{M}} b \frac{I_{\mathrm{vS}}}{N_{\mathrm{H}}} S_{\mathrm{H}}-\left(\tau_{\mathrm{M} 1}+\mu_{\mathrm{H}}+\alpha_{\mathrm{MS}}+\delta_{\mathrm{MS} 1}\right) I_{\mathrm{US}}^{\mathrm{M}}-\frac{B_{\mathrm{C}} \varphi}{\kappa+B_{\mathrm{C}}} I_{\mathrm{Us}}^{\mathrm{M}}+\frac{\beta_{\mathrm{v}} b I_{\mathrm{vs}}}{N_{\mathrm{H}}} R_{\mathrm{C}}+h u_{1}(t) \theta_{1}(1-f) I_{\mathrm{USC}}^{\mathrm{M}} \\
& \frac{d I_{\mathrm{TS}}^{\mathrm{M}}}{d t}=\tau_{\mathrm{M} 1} I_{\mathrm{US}}^{\mathrm{M}}-\left(\sigma_{\mathrm{M} 1}+\mu_{\mathrm{H}}+\alpha_{\mathrm{MT}}+\phi_{1} \delta_{\mathrm{MS} 1}\right) I_{\mathrm{TS}}^{\mathrm{M}}-\frac{B_{\mathrm{c}} \varphi}{\kappa+B_{\mathrm{C}}} I_{\mathrm{TS}}^{\mathrm{M}}+h u_{2}(t) \theta_{2}(1-f) I_{\mathrm{TSC}}^{\mathrm{M}} \\
& \frac{d I_{\mathrm{R}}^{\mathrm{M}}}{d t}=\sigma_{\mathrm{M} 1} I_{\mathrm{TS}}^{\mathrm{M}}+\frac{\beta_{\mathrm{M}} b \eta_{\mathrm{R}} I_{\mathrm{VR}}}{N_{\mathrm{H}}} S_{\mathrm{H}}-\left(\mu_{\mathrm{H}}+\alpha_{\mathrm{MR}}+\delta_{\mathrm{MR} 1}\right) I_{\mathrm{R}}^{\mathrm{M}}-\frac{B_{\mathrm{C}} \varphi}{\kappa+B_{\mathrm{C}}} I_{\mathrm{R}}^{\mathrm{M}}+\frac{\beta_{\mathrm{M}} b \eta_{\mathrm{R}} I_{\mathrm{VR}}}{N_{\mathrm{H}}} R_{\mathrm{C}}+h u_{3}(t) \theta_{3}(1-f) I_{\mathrm{RC}}^{\mathrm{M}} \\
& \frac{d R_{\mathrm{M}}}{d t}=\alpha_{\mathrm{MS}} I_{\mathrm{US}}^{\mathrm{M}}+\alpha_{\mathrm{MT}} I_{\mathrm{TS}}^{\mathrm{M}}+\alpha_{\mathrm{MR}} I_{\mathrm{R}}^{\mathrm{M}}-\left(\mu+\omega_{\mathrm{M}}\right) R_{\mathrm{M}}-\frac{B_{\mathrm{c}} \varphi}{\kappa+B_{\mathrm{C}}} R_{\mathrm{M}} \\
& \frac{d I_{\mathrm{C}}}{d t}=\frac{\beta_{\mathrm{C}} \varphi}{\kappa+B_{\mathrm{C}}}\left(S_{\mathrm{H}}+R_{\mathrm{M}}\right)-\left(\delta_{\mathrm{C} 1}+\mu_{\mathrm{H}}+\gamma_{\mathrm{C}}\right) I_{\mathrm{C}}-\frac{\beta_{\mathrm{v}} b I_{\mathrm{vS}}}{N_{\mathrm{H}}} I_{\mathrm{C}}-\frac{\beta_{\mathrm{M}} b \eta_{\mathrm{R}} I_{\mathrm{VR}}}{N_{\mathrm{H}}} I_{\mathrm{C}} \\
& +f u_{1}(t) \theta_{1} I_{\mathrm{usC}}^{\mathrm{M}}+f u_{2}(t) \theta_{2} I_{\mathrm{TSC}}^{\mathrm{M}}+f u_{3}(t) \theta_{3} I_{\mathrm{RC}}^{\mathrm{M}} \\
& \frac{d R_{\mathrm{C}}}{d t}=\gamma_{\mathrm{C}} I_{\mathrm{C}}-\left(\mu_{\mathrm{H}}+\omega_{\mathrm{C}}\right) R_{\mathrm{C}}-\frac{\beta_{\mathrm{M}} b\left(I_{\mathrm{vS}}+\eta_{\mathrm{R}} I_{\mathrm{VR}}\right)}{N_{\mathrm{H}}} R_{\mathrm{C}} \\
& \frac{d B_{\mathrm{C}}}{d t}=\rho_{\mathrm{C}} I_{\mathrm{C}}+\rho_{\mathrm{C}}\left(I_{\mathrm{USC}}^{\mathrm{M}}+\sigma_{\mathrm{T}} I_{\mathrm{TSC}}^{\mathrm{M}}+\sigma_{\mathrm{R}} I_{\mathrm{RC}}^{\mathrm{M}}\right)-\mu_{\mathrm{B}} B_{\mathrm{C}} \\
& \frac{d I_{\mathrm{vSC}}^{\mathrm{M}}}{d t}=\frac{B_{\mathrm{c}} \varphi}{\kappa+B_{\mathrm{C}}} I_{\mathrm{us}}^{\mathrm{M}}+\frac{\beta_{\mathrm{M}} b I_{\mathrm{vs}}}{N_{\mathrm{H}}} I_{\mathrm{C}}-\mu_{\mathrm{H}} I_{\mathrm{usC}}^{\mathrm{M}}-\delta_{\mathrm{Ms} 2} I_{\mathrm{UsC}}^{\mathrm{M}}-\delta_{\mathrm{C} 2} I_{\mathrm{usC}}^{\mathrm{M}}-\tau_{\mathrm{M} 2} I_{\mathrm{usC}}^{\mathrm{M}}-u_{1}(t) \theta_{1} I_{\mathrm{usC}}^{\mathrm{M}} \\
& \frac{d I_{\mathrm{TSC}}^{\mathrm{M}}}{d t}=\frac{B_{\mathrm{c}} \varphi}{\kappa+B_{\mathrm{C}}} I_{\mathrm{TS}}^{\mathrm{M}}+\tau_{\mathrm{M} 2} I_{\mathrm{USC}}^{\mathrm{M}}-\mu_{\mathrm{H}} I_{\mathrm{TSC}}^{\mathrm{M}}-\phi_{2} \delta_{\mathrm{MS} 2} I_{\mathrm{TSC}}^{\mathrm{M}}-u_{2}(t) \theta_{2} I_{\mathrm{TSC}}^{\mathrm{M}}-\sigma_{\mathrm{M} 2} I_{\mathrm{TSC}}^{\mathrm{M}}-\delta_{\mathrm{C} 2} I_{\mathrm{TSC}}^{\mathrm{M}} \\
& \frac{d I_{\mathrm{RC}}^{\mathrm{M}}}{d t}=\sigma_{\mathrm{M} 2} I_{\mathrm{TSC}}^{\mathrm{M}}+\frac{B_{\mathrm{C}} \varphi}{\kappa+B_{\mathrm{C}}} I_{\mathrm{R}}^{\mathrm{M}}+\frac{\beta_{\mathrm{v}} b \eta_{\mathrm{R}} I_{\mathrm{R}}^{\mathrm{M}}}{N_{\mathrm{H}}} I_{\mathrm{C}}-\mu_{\mathrm{H}} I_{\mathrm{RC}}^{\mathrm{M}}-\delta_{\mathrm{MR} 2} I_{\mathrm{RC}}^{\mathrm{M}}-\delta_{\mathrm{C} 2} I_{\mathrm{RC}}^{\mathrm{M}}-u_{3}(t) \theta_{3} I_{\mathrm{RC}}^{\mathrm{M}} \\
& \frac{d R_{\mathrm{MC}}}{d t}=u_{1}(t) \theta_{1}(1-f)(1-h) I_{\mathrm{usC}}^{\mathrm{M}}+u_{2}(t) \theta_{2}(1-f)(1-h) I_{\mathrm{TSC}}^{\mathrm{M}}+u_{3}(t) \theta_{3}(1-f)(1-h) I_{\mathrm{RC}}^{\mathrm{M}}-\left(\omega_{\mathrm{MC}}+\mu_{\mathrm{H}}\right) R_{\mathrm{MC}} \\
& \frac{d S_{\mathrm{v}}}{d t}=\pi_{\mathrm{v}}-\frac{\beta_{\mathrm{v}} b\left(I_{\mathrm{US}}^{\mathrm{M}}+\eta_{\mathrm{T}} I_{\mathrm{TS}}^{\mathrm{M}}+\eta_{\mathrm{R}} I_{\mathrm{R}}^{\mathrm{M}}\right)}{N_{\mathrm{H}}} S_{\mathrm{v}}-\frac{\beta_{\mathrm{v}} b\left(I_{\mathrm{USC}}^{\mathrm{M}}+\eta_{\mathrm{T}} I_{\mathrm{TSC}}^{\mathrm{M}}+\eta_{\mathrm{R}} I_{\mathrm{RC}}^{\mathrm{M}}\right)}{N_{\mathrm{H}}} S_{\mathrm{v}}-\mu_{\mathrm{v}} S_{\mathrm{V}} \\
& \frac{d I_{\mathrm{vS}}}{d t}=\frac{\beta_{\mathrm{v}} b\left(I_{\mathrm{US}}^{\mathrm{M}}+\eta_{\mathrm{T}} I_{\mathrm{TS}}^{\mathrm{M}}\right)}{N_{\mathrm{H}}} S_{\mathrm{v}}+\frac{\beta_{\mathrm{v}} b\left(I_{\mathrm{USC}}^{\mathrm{M}}+\eta_{\mathrm{T}} I_{\mathrm{TSC}}^{\mathrm{M}}\right)}{N_{\mathrm{H}}} S_{\mathrm{v}}-\mu_{\mathrm{v}} I_{\mathrm{vS}} \\
& \frac{d I_{\mathrm{VR}}}{d t}=\frac{\beta_{\mathrm{v}} b \eta_{\mathrm{R}} I_{\mathrm{R}}^{\mathrm{M}}}{N_{\mathrm{H}}} S_{\mathrm{v}}+\frac{\beta_{\mathrm{v}} b \eta_{\mathrm{R}} I_{\mathrm{RC}}^{\mathrm{M}}}{N_{\mathrm{H}}} S_{\mathrm{V}}-\mu_{\mathrm{v}} I_{\mathrm{VR}}
\end{aligned}
$$

The control functions, $u_{1}(t), u_{2}(t)$, and $u_{3}(t)$ are bounded, Lebesgue integrable functions. The control $u_{1}(t)$ represents treatment efforts for co-infected individuals in $I_{\text {usc }}^{\mathrm{M}}$ compartment. $u_{2}(t)$ represents treatment efforts for co-infected individuals in $I_{\mathrm{TSC}}^{\mathrm{M}}$ compartment. The control $u_{3}(t)$ represents treatment efforts for co-infected individuals in $I_{\mathrm{RC}}^{\mathrm{M}}$ compartment. The controls $u_{1}, u_{2}, u_{3}$ satisfies $0 \leq u_{1}, u_{2}, u_{3} \leq 1$. The optimal control system examines scenarios where the number of co-infected cases and the cost of implementing the controls $u_{1}(t), u_{2}(t)$, and $u_{3}(t)$ are minimized subject to the state system (11). For this, we consider the objective functional

$$
J\left[u_{1}, u_{2}, u_{3}\right]=\int_{0}^{T}\left[I_{\mathrm{USC}}^{\mathrm{M}}(t)+I_{\mathrm{TSC}}^{\mathrm{M}}(t)+I_{\mathrm{RC}}^{\mathrm{M}}(t)+\frac{B_{1}}{2} u_{1}^{2}+\frac{B_{2}}{2} u_{2}^{2}+\frac{B_{3}}{2} u_{3}^{2}\right] d t
$$

$T$ is the final time. We seek to find an optimal control, $u_{1}^{*}, u_{2}^{*}, u_{3}^{*}$, such that

$$
J\left(u_{1}^{*}, u_{2}^{*}, u_{3}^{*}\right)=\min \left\{J\left(u_{1}^{*}, u_{2}^{*}, u_{3}^{*}\right) \mid u_{1}, u_{2}, u_{3} \in U\right\}
$$

where $U=\left\{\left(u_{1}^{*}, u_{2}^{*}, u_{3}^{*}\right)\right\}$ such that $u_{1}^{*}, u_{2}^{*}, u_{3}^{*}$ are measurable with $0 \leq u_{1}^{*} \leq 1,0 \leq u_{2}^{*} \leq 1,0 \leq u_{3}^{*} \leq 1$, for $t \in[0, T]$ is the control set. The Pontryagin's Maximum Principle [28] gives the necessary conditions which an optimal control pair must satisfy. This principle transforms (11), (12) and (13) into a problem 
of minimizing a Hamiltonian, $\mathcal{H}$, pointwisely with regards to the control functions, $u_{1}, u_{2}, u_{3}$ :

$$
\begin{aligned}
& \mathcal{H}=I_{\mathrm{UsC}}^{\mathrm{M}}(t)+I_{\mathrm{TSC}}^{\mathrm{M}}(t)+I_{\mathrm{RC}}^{\mathrm{M}}(t)+\frac{B_{1}}{2} u_{1}^{2}+\frac{B_{2}}{2} u_{2}^{2}+\frac{B_{3}}{2} u_{3}^{2}+\frac{B_{4}}{2} u_{4}^{2} \\
& +\lambda_{S_{\mathrm{H}}}\left[\pi_{\mathrm{H}}-\frac{\beta_{\mathrm{M}} b\left(I_{\mathrm{VS}}+\eta_{\mathrm{R}} I_{\mathrm{VR}}\right)}{N_{\mathrm{H}}} S_{\mathrm{H}}-\frac{B_{\mathrm{C}} \varphi}{\kappa+B_{\mathrm{C}}} S_{\mathrm{H}}-\mu_{\mathrm{H}} S_{\mathrm{H}}+\omega_{\mathrm{M}} R_{\mathrm{M}}+\omega_{\mathrm{C}} R_{\mathrm{C}}+\omega_{\mathrm{MC}} R_{\mathrm{MC}}\right] \\
& +\lambda_{I_{\mathrm{US}}^{\mathrm{M}}}\left[\beta_{\mathrm{M}} b \frac{I_{\mathrm{vS}}}{N_{\mathrm{H}}} S_{\mathrm{H}}-\left(\tau_{\mathrm{M} 1}+\mu_{\mathrm{H}}+\alpha_{\mathrm{MS}}+\delta_{\mathrm{MS} 1}\right) I_{\mathrm{US}}^{\mathrm{M}}-\frac{B_{\mathrm{C}} \varphi}{\kappa+B_{\mathrm{C}}} I_{\mathrm{US}}^{\mathrm{M}}+\frac{\beta_{\mathrm{v}} b I_{\mathrm{vs}}}{N_{\mathrm{H}}} R_{\mathrm{C}}+h u_{1}(t) \theta_{1}(1-f) I_{\mathrm{UsC}}^{\mathrm{M}}\right] \\
& +\lambda_{I_{\mathrm{TS}}^{\mathrm{M}}}\left[\tau_{\mathrm{M} 1} I_{\mathrm{US}}^{\mathrm{M}}-\left(\sigma_{\mathrm{M} 1}+\mu_{\mathrm{H}}+\alpha_{\mathrm{MT}}+\phi_{1} \delta_{\mathrm{MS} 1}\right) I_{\mathrm{Ts}}^{\mathrm{M}}-\frac{B_{\mathrm{c}} \varphi}{\kappa+B_{\mathrm{C}}} I_{\mathrm{TS}}^{\mathrm{M}}+h u_{2}(t) \theta_{2}(1-f) I_{\mathrm{TSC}}^{\mathrm{M}}\right] \\
& +\lambda_{I_{\mathrm{R}}^{\mathrm{M}}}\left[\sigma_{\mathrm{M} 1} I_{\mathrm{TS}}^{\mathrm{M}}+\frac{\beta_{\mathrm{M}} b \eta_{\mathrm{R}} I_{\mathrm{VR}}}{N_{\mathrm{H}}} S_{\mathrm{H}}-\left(\mu_{\mathrm{H}}+\alpha_{\mathrm{MR}}+\delta_{\mathrm{MR} 1}\right) I_{\mathrm{R}}^{\mathrm{M}}-\frac{B_{\mathrm{C}} \varphi}{\kappa+B_{\mathrm{C}}} I_{\mathrm{R}}^{\mathrm{M}}+\frac{\beta_{\mathrm{M}} b \eta_{\mathrm{R}} I_{\mathrm{VR}}}{N_{\mathrm{H}}} R_{\mathrm{C}}+h u_{3}(t) \theta_{3}(1-f) I_{\mathrm{RC}}^{\mathrm{M}}\right] \\
& +\lambda_{R_{\mathrm{M}}}\left[\alpha_{\mathrm{MS}} I_{\mathrm{US}}^{\mathrm{M}}+\alpha_{\mathrm{MT}} I_{\mathrm{TS}}^{\mathrm{M}}+\alpha_{\mathrm{MR}} I_{\mathrm{R}}^{\mathrm{M}}-\left(\mu+\omega_{\mathrm{M}}\right) R_{\mathrm{M}}-\frac{B_{\mathrm{C}} \varphi}{\kappa+B_{\mathrm{C}}} R_{\mathrm{M}}\right] \\
& +\lambda_{I_{\mathrm{C}}}\left[\frac{\beta_{\mathrm{c}} \varphi}{\kappa+B_{\mathrm{C}}}\left(S_{\mathrm{H}}+R_{\mathrm{M}}\right)-\left(\delta_{\mathrm{c} 1}+\mu_{\mathrm{H}}+\gamma_{\mathrm{C}}\right) I_{\mathrm{C}}-\frac{\beta_{\mathrm{v}} b I_{\mathrm{vS}}}{N_{\mathrm{H}}} I_{\mathrm{C}}-\frac{\beta_{\mathrm{M}} b \eta_{\mathrm{R}} I_{\mathrm{VR}}}{N_{\mathrm{H}}} I_{\mathrm{C}}+f u_{1}(t) \theta_{1} I_{\mathrm{USC}}^{\mathrm{M}}+f u_{2}(t) \theta_{2} I_{\mathrm{TSC}}^{\mathrm{M}}+f u_{3}(t) \theta_{3} I_{\mathrm{rC}}^{\mathrm{M}}\right] \\
& +\lambda_{R_{\mathrm{C}}}\left[\gamma_{\mathrm{C}} I_{\mathrm{C}}-\left(\mu_{\mathrm{H}}+\omega_{\mathrm{C}}\right) R_{\mathrm{C}}-\frac{\beta_{\mathrm{M}} b\left(I_{\mathrm{vS}}+\eta_{\mathrm{R}} I_{\mathrm{VR}}\right)}{N_{\mathrm{H}}} R_{\mathrm{C}}\right] \\
& +\lambda_{B_{\mathrm{C}}}\left[\rho_{\mathrm{c}} I_{\mathrm{C}}+\rho_{\mathrm{C}}\left(I_{\mathrm{USC}}^{\mathrm{M}}+\sigma_{\mathrm{T}} I_{\mathrm{TSC}}^{\mathrm{M}}+\sigma_{\mathrm{R}} I_{\mathrm{RC}}^{\mathrm{M}}\right)-\mu_{\mathrm{B}} B_{\mathrm{C}}\right] \\
& +\lambda_{I_{\mathrm{USC}}^{\mathrm{M}}}\left[\frac{B_{\mathrm{c}} \varphi}{\kappa+B_{\mathrm{C}}} I_{\mathrm{US}}^{\mathrm{M}}+\frac{\beta_{\mathrm{M}} b I_{\mathrm{vs}}}{N_{\mathrm{H}}} I_{\mathrm{C}}-\mu_{\mathrm{H}} I_{\mathrm{UsC}}^{\mathrm{M}}-\delta_{\mathrm{Ms} 2} I_{\mathrm{UsC}}^{\mathrm{M}}-\delta_{\mathrm{C} 2} I_{\mathrm{usC}}^{\mathrm{M}}-\tau_{\mathrm{M} 2} I_{\mathrm{UsC}}^{\mathrm{M}}-u_{1}(t) \theta_{1} I_{\mathrm{USC}}^{\mathrm{M}}\right] \\
& +\lambda_{I_{\mathrm{TSC}}^{\mathrm{M}}}\left[\frac{B_{\mathrm{C}} \varphi}{\kappa+B_{\mathrm{C}}} I_{\mathrm{TS}}^{\mathrm{M}}+\tau_{\mathrm{M} 2} I_{\mathrm{usC}}^{\mathrm{M}}-\mu_{\mathrm{H}} I_{\mathrm{TSC}}^{\mathrm{M}}-\phi_{2} \delta_{\mathrm{MS} 2} I_{\mathrm{TSC}}^{\mathrm{M}}-u_{2}(t) \theta_{2} I_{\mathrm{TSC}}^{\mathrm{M}}-\sigma_{\mathrm{M} 2} I_{\mathrm{TSC}}^{\mathrm{M}}-\delta_{\mathrm{C} 2} I_{\mathrm{TSC}}^{\mathrm{M}}\right] \\
& +\lambda_{I_{\mathrm{RC}}^{\mathrm{M}}}\left[\sigma_{\mathrm{M} 2} I_{\mathrm{TSC}}^{\mathrm{M}}+\frac{B_{\mathrm{c}} \varphi}{\kappa+B_{\mathrm{C}}} I_{\mathrm{R}}^{\mathrm{M}}+\frac{\beta_{\mathrm{v}} b \eta_{\mathrm{R}} I_{\mathrm{R}}^{\mathrm{M}}}{N_{\mathrm{H}}} I_{\mathrm{C}}-\mu_{\mathrm{H}} I_{\mathrm{RC}}^{\mathrm{M}}-\delta_{\mathrm{MR} 2} I_{\mathrm{RC}}^{\mathrm{M}}-\delta_{\mathrm{C} 2} I_{\mathrm{RC}}^{\mathrm{M}}-u_{3}(t) \theta_{3} I_{\mathrm{RC}}^{\mathrm{M}}\right] \\
& +\lambda_{R_{\mathrm{MC}}}\left[u_{1}(t) \theta_{1}(1-f)(1-h) I_{\mathrm{USC}}^{\mathrm{M}}+u_{2}(t) \theta_{2}(1-f)(1-h) I_{\mathrm{TSC}}^{\mathrm{M}}+u_{3}(t) \theta_{3}(1-f)(1-h) I_{\mathrm{RC}}^{\mathrm{M}}-\left(\omega_{\mathrm{MC}}+\mu_{\mathrm{H}}\right) R_{\mathrm{MC}}\right] \\
& +\lambda_{S_{\mathrm{v}}}\left[\pi_{\mathrm{v}}-\frac{\beta_{\mathrm{v}} b\left(I_{\mathrm{US}}^{\mathrm{M}}+\eta_{\mathrm{T}} I_{\mathrm{TS}}^{\mathrm{M}}+\eta_{\mathrm{R}} I_{\mathrm{R}}^{\mathrm{M}}\right)}{N_{\mathrm{H}}} S_{\mathrm{v}}-\frac{\beta_{\mathrm{v}} b\left(I_{\mathrm{USC}}^{\mathrm{M}}+\eta_{\mathrm{T}} I_{\mathrm{TSC}}^{\mathrm{M}}+\eta_{\mathrm{R}} I_{\mathrm{RC}}^{\mathrm{M}}\right)}{N_{\mathrm{H}}} S_{\mathrm{v}}-\mu_{\mathrm{v}} S_{\mathrm{v}}\right] \\
& +\lambda_{I_{\mathrm{VS}}}\left[\frac{\beta_{\mathrm{v}} b\left(I_{\mathrm{US}}^{\mathrm{M}}+\eta_{\mathrm{T}} I_{\mathrm{TS}}^{\mathrm{M}}\right)}{N_{\mathrm{H}}} S_{\mathrm{v}}+\frac{\beta_{\mathrm{v}} b\left(I_{\mathrm{USC}}^{\mathrm{M}}+\eta_{\mathrm{T}} I_{\mathrm{TSC}}^{\mathrm{M}}\right)}{N_{\mathrm{H}}} S_{\mathrm{v}}-\mu_{\mathrm{v}} I_{\mathrm{vS}}\right] \\
& +\lambda_{I_{\mathrm{VR}}}\left[\frac{\beta_{\mathrm{v}} b \eta_{\mathrm{R}} I_{\mathrm{R}}^{\mathrm{M}}}{N_{\mathrm{H}}} S_{\mathrm{v}}+\frac{\beta_{\mathrm{V}} b \eta_{\mathrm{R}} I_{\mathrm{RC}}^{\mathrm{M}}}{N_{\mathrm{H}}} S_{\mathrm{v}}-\mu_{\mathrm{v}} I_{\mathrm{VR}}\right]
\end{aligned}
$$

Theorem 4.1 For an optimal control set $u_{1}, u_{2}, u_{3}$ that minimizes $J$ over $U$, there are adjoint variables, $\lambda_{1}, \lambda_{2}, \ldots, \lambda_{15}$ satisfying

$$
-\frac{\partial \lambda_{i}}{\partial t}=\frac{\partial \mathcal{H}}{\partial i}
$$

and with transversality conditions

$$
\lambda_{i}\left(t_{f}\right)=0, \quad \text { where, } \quad i=S_{\mathrm{H}}, I_{\mathrm{Us}}^{\mathrm{M}}, I_{\mathrm{Ts}}^{\mathrm{M}}, I_{\mathrm{R}}^{\mathrm{M}}, R_{\mathrm{M}}, I_{\mathrm{C}}, R_{\mathrm{c}}, B_{\mathrm{C}}, I_{\mathrm{usc}}^{\mathrm{M}}, I_{\mathrm{TsC}}^{\mathrm{M}}, I_{\mathrm{RC}}^{\mathrm{M}}, R_{\mathrm{vS}}, S_{\mathrm{v}}, I_{\mathrm{vs}}, I_{\mathrm{vR}} .
$$

Furthermore,

$$
\begin{aligned}
& u_{1}^{*}=\max \left\{0, \min \left(1, \frac{\left[\left(\lambda_{9}-f \lambda_{6}\right)-(1-f)(1-h) \lambda_{12}-h(1-f) \lambda_{2}\right] \theta_{1} I_{\mathrm{USC}}^{\mathrm{M}}}{B_{1}}\right)\right\}, \\
& u_{2}^{*}=\max \left\{0, \min \left(1, \frac{\left[\left(\lambda_{10}-f \lambda_{6}\right)-(1-f)(1-h) \lambda_{12}-h(1-f) \lambda_{3}\right] \theta_{2} I_{\mathrm{TSC}}^{\mathrm{M}}}{B_{2}}\right)\right\}, \\
& u_{3}^{*}=\max \left\{0, \min \left(1, \frac{\left[\left(\lambda_{1} 1-f \lambda_{6}\right)-(1-f)(1-h) \lambda_{12}-h(1-f) \lambda_{4}\right] \theta_{3} I_{\mathrm{RC}}^{\mathrm{M}}}{B_{3}},\right)\right\},
\end{aligned}
$$

\section{Proof of Theorem 4.1}

Suppose $U^{*}=\left(u_{1}^{*}, u_{2}^{*}, u_{3}^{*}\right)$ is an optimal control and $S_{\mathrm{H}}, I_{\mathrm{Us}}^{\mathrm{M}}, I_{\mathrm{Ts}}^{\mathrm{M}}, I_{\mathrm{R}}^{\mathrm{M}}, R_{\mathrm{M}}, I_{\mathrm{C}}, B_{\mathrm{C}}, I_{\mathrm{USC}}^{\mathrm{M}}, I_{\mathrm{TSC}}^{\mathrm{M}}, I_{\mathrm{RC}}^{\mathrm{M}}, R_{\mathrm{US}}, S_{\mathrm{v}}, I_{\mathrm{vs}}, I_{\mathrm{VR}}$. are the corresponding state solutions. Applying the Pontryagin's Maximum Principle [28], there exist 
adjoint variables satisfying:

$$
\begin{aligned}
& -\frac{d \lambda_{S_{\mathrm{H}}}}{d t}=\frac{\partial \mathcal{H}}{\partial S_{\mathrm{H}}}, \quad \lambda_{S_{\mathrm{H}}}\left(t_{f}\right)=0, \quad-\frac{d \lambda_{I_{\mathrm{US}}^{\mathrm{M}}}}{d t}=\frac{\partial \mathcal{H}}{\partial I_{\mathrm{US}}^{\mathrm{M}}}, \quad \lambda_{I_{\mathrm{US}}^{\mathrm{M}}}\left(t_{f}\right)=0, \quad-\frac{d \lambda_{I_{\mathrm{TS}}^{\mathrm{M}}}}{d t}=\frac{\partial \mathcal{H}}{\partial I_{\mathrm{TS}}^{\mathrm{M}}}, \quad \lambda_{I_{\mathrm{TS}}^{\mathrm{M}}}\left(t_{f}\right)=0, \\
& -\frac{d \lambda_{I_{\mathrm{R}}^{\mathrm{M}}}}{d t}=\frac{\partial \mathcal{H}}{\partial I_{\mathrm{R}}^{\mathrm{M}}}, \quad \lambda_{I_{\mathrm{R}}^{\mathrm{M}}}\left(t_{f}\right)=0,-\frac{d \lambda_{R_{\mathrm{M}}}}{d t}=\frac{\partial \mathcal{H}}{\partial R_{\mathrm{M}}}, \quad \lambda_{R_{\mathrm{M}}}\left(t_{f}\right)=0, \quad-\frac{d \lambda_{I_{\mathrm{C}}}}{d t}=\frac{\partial \mathcal{H}}{\partial I_{\mathrm{C}}}, \quad \lambda_{I_{\mathrm{C}}}\left(t_{f}\right)=0, \\
& -\frac{d \lambda_{I_{\mathrm{C}}}}{d t}=\frac{\partial \mathcal{H}}{\partial I_{\mathrm{C}}}, \quad \lambda_{I_{\mathrm{C}}}\left(t_{f}\right)=0, \quad-\frac{d \lambda_{B_{\mathrm{C}}}}{d t}=\frac{\partial \mathcal{H}}{\partial B_{\mathrm{C}}}, \quad \lambda_{B_{\mathrm{C}}}\left(t_{f}\right)=0 \\
& -\frac{d \lambda_{I_{\mathrm{USC}}^{\mathrm{M}}}}{d t}=\frac{\partial \mathcal{H}}{\partial I_{\mathrm{USC}}^{\mathrm{M}}}, \quad \lambda_{I_{\mathrm{USC}}}\left(t_{f}\right)=0, \quad-\frac{d \lambda_{I_{\mathrm{TSC}}^{\mathrm{M}}}}{d t}=\frac{\partial \mathcal{H}}{\partial I_{\mathrm{TSC}}^{\mathrm{M}}}, \quad \lambda_{I_{\mathrm{TSC}}^{\mathrm{M}}}\left(t_{f}\right)=0 \\
& -\frac{d \lambda_{I_{\mathrm{RC}}^{\mathrm{M}}}}{d t}=\frac{\partial \mathcal{H}}{\partial I_{\mathrm{RC}}^{\mathrm{M}}}, \quad \lambda_{I_{\mathrm{RC}}^{\mathrm{M}}}\left(t_{f}\right)=0, \quad-\frac{d \lambda_{R_{\mathrm{MC}}}}{d t}=\frac{\partial \mathcal{H}}{\partial R_{\mathrm{MC}}}, \quad \lambda_{R_{\mathrm{MC}}}\left(t_{f}\right)=0 \\
& -\frac{d \lambda_{S_{\mathrm{V}}}}{d t}=\frac{\partial \mathcal{H}}{\partial S_{\mathrm{V}}}, \quad \lambda_{S_{\mathrm{V}}}\left(t_{f}\right)=0, \quad-\frac{d \lambda_{I_{\mathrm{Vs}}}}{d t}=\frac{\partial \mathcal{H}}{\partial I_{\mathrm{Vs}}}, \quad \lambda_{I_{\mathrm{VS}}}\left(t_{f}\right)=0, \quad-\frac{d \lambda_{I_{\mathrm{VR}}}}{d t}=\frac{\partial \mathcal{H}}{\partial I_{\mathrm{VR}}}, \quad \lambda_{I_{\mathrm{VR}}}\left(t_{f}\right)=0
\end{aligned}
$$

with transversality conditions;

$S_{\mathrm{H}}=I_{\mathrm{US}}^{\mathrm{M}}=I_{\mathrm{TS}}^{\mathrm{M}}=I_{\mathrm{R}}^{\mathrm{M}}=R_{\mathrm{M}}=I_{\mathrm{C}}=B_{\mathrm{C}}=I_{\mathrm{USC}}^{\mathrm{M}}=I_{\mathrm{TSC}}^{\mathrm{M}}=I_{\mathrm{RC}}^{\mathrm{M}}=R_{\mathrm{US}}=S_{\mathrm{v}}=I_{\mathrm{vs}}=I_{\mathrm{vR}}=0$ We can determine the behaviour of the control by differentiating the Hamiltonian, $\mathcal{H}$ with respect to the $\operatorname{controls}\left(u_{1}, u_{2}, u_{3}, u_{4}\right)$ at $t$. On the interior of the control set, where $0<u_{j}<1$ for all $(j=1,2,3,4)$, we obtain

$$
\begin{aligned}
& 0=\frac{\partial \mathcal{H}}{\partial u_{1}}=B_{1} u_{1}^{*}-\theta_{1} I_{\mathrm{USC}}^{\mathrm{M}}\left[\lambda_{9}-f \lambda_{6}-(1-f)(1-h) \lambda_{12}-h(1-h) \lambda_{2}\right], \\
& 0=\frac{\partial \mathcal{H}}{\partial u_{2}}=B_{2} u_{2}^{*}-I_{\mathrm{TSC}}^{\mathrm{M}} \theta_{2}\left[\lambda_{10}-f \lambda_{6}-(1-f)(1-h) \lambda_{12}-h(1-f) \lambda_{3}\right], \\
& 0=\frac{\partial \mathcal{H}}{\partial u_{3}}=B_{3} u_{3}^{*}-I_{\mathrm{RC}}^{\mathrm{M}} \theta_{3}\left[\lambda_{11}-f \lambda_{6}-(1-f)(1-h) \lambda_{12}-h(1-f) \lambda_{4}\right],
\end{aligned}
$$

Therefore, we have that 29 ]

$$
\begin{gathered}
u_{1}^{*}=\frac{\left[\left(\lambda_{9}-f \lambda_{6}\right)-(1-f)(1-h) \lambda_{12}-h(1-f) \lambda_{2}\right] \theta_{1} I_{\mathrm{Usc}}^{\mathrm{M}}}{B_{1}}, \\
u_{2}^{*}=\frac{\left[\left(\lambda_{10}-f \lambda_{6}\right)-(1-f)(1-h) \lambda_{12}-h(1-f) \lambda_{3}\right] \theta_{2} I_{\mathrm{rsc}}^{\mathrm{M}}}{B_{2}}, \\
u_{3}^{*}=\frac{\left[\left(\lambda_{1} 1-f \lambda_{6}\right)-(1-f)(1-h) \lambda_{12}-h(1-f) \lambda_{4}\right] \theta_{3} I_{\mathrm{RC}}^{\mathrm{M}}}{B_{3}}, \\
u_{1}^{*}=\max \left\{0, \min \left(1, \frac{\left[\left(\lambda_{9}-f \lambda_{6}\right)-(1-f)(1-h) \lambda_{12}-h(1-f) \lambda_{2}\right] \theta_{1} I_{\mathrm{USC}}^{\mathrm{M}}}{B_{1}}\right)\right\}, \\
u_{2}^{*}=\max \left\{0, \min \left(1, \frac{\left[\left(\lambda_{10}-f \lambda_{6}\right)-(1-f)(1-h) \lambda_{12}-h(1-f) \lambda_{3}\right] \theta_{2} I_{\mathrm{TSC}}^{\mathrm{M}}}{B_{2}}\right)\right\}, \\
u_{3}^{*}=\max \left\{0, \min \left(1, \frac{\left[\left(\lambda_{1} 1-f \lambda_{6}\right)-(1-f)(1-h) \lambda_{12}-h(1-f) \lambda_{4}\right] \theta_{3} I_{\mathrm{RC}}^{\mathrm{M}}}{B_{3}},\right)\right\},
\end{gathered}
$$

\section{$5 \quad$ Numerical simulations}

Figure 2 shows the simulation of the total number of individuals untreated of sensitive strain and coinfected with cholera, when there is no drug resistance. it is observed that a total of 1,590 co-infected cases were averted. However as presented in Figure 3 when there is drug resistance, a total of 237 coinfection cases were averted. figure 4 shows the simulations of the total number of individuals treated of sensitive malaria strain and co-infected with cholera. It is observed that a total of 2,527 co-infection cases were prevented, when there is no resistance. However, when there is malaria drug resistance, as depicted figure 5, a total of 116 co-infection cases were averted. Figure 6 depicts the simulations of the total number of individuals co-infected with resistant malaria and cholera. It is seen that in the absence of malaria drug resistance, the treatment control applied prevented a total of 36,350 co-infection cases. However, in the presence of malaria drug resistance, as shown Figure 7 a total of 40,590 co-infection cases were averted when the treatment control is applied. It is imperative to note that in the presence of 
medRxiv preprint doi: https://doi.org/10.1101/2020.08.18.20177329; this version posted September 10, 2020. The copyright holder for this preprint (which was not certified by peer review) is the author/funder, who has granted medRxiv a license to display the preprint in

It is made available under a CC-BY-NC-ND 4.0 International license .

treatment controls, more co-infection cases were averted when there is drug resistance, than whe there is no drug resustance, for co-infected individuals in compartments $I_{\mathrm{usc}}^{\mathrm{M}}$ and $I_{\mathrm{Tsc}}^{\mathrm{M}}$. However, it is observed that for co-infected individuals in compartment $I_{\mathrm{RC}}^{\mathrm{M}}$, more co-infection cases were averted in the presence of malaria drug resistance than when there is no drug resistance, when treatment controls are applied in both cases. This points to the fact that malaria drug resistance can greatly influence the co-infection cases averted, even in the presence of treatment controls for co-infected individuals.

Table 2: Description of parameters in the model (2).

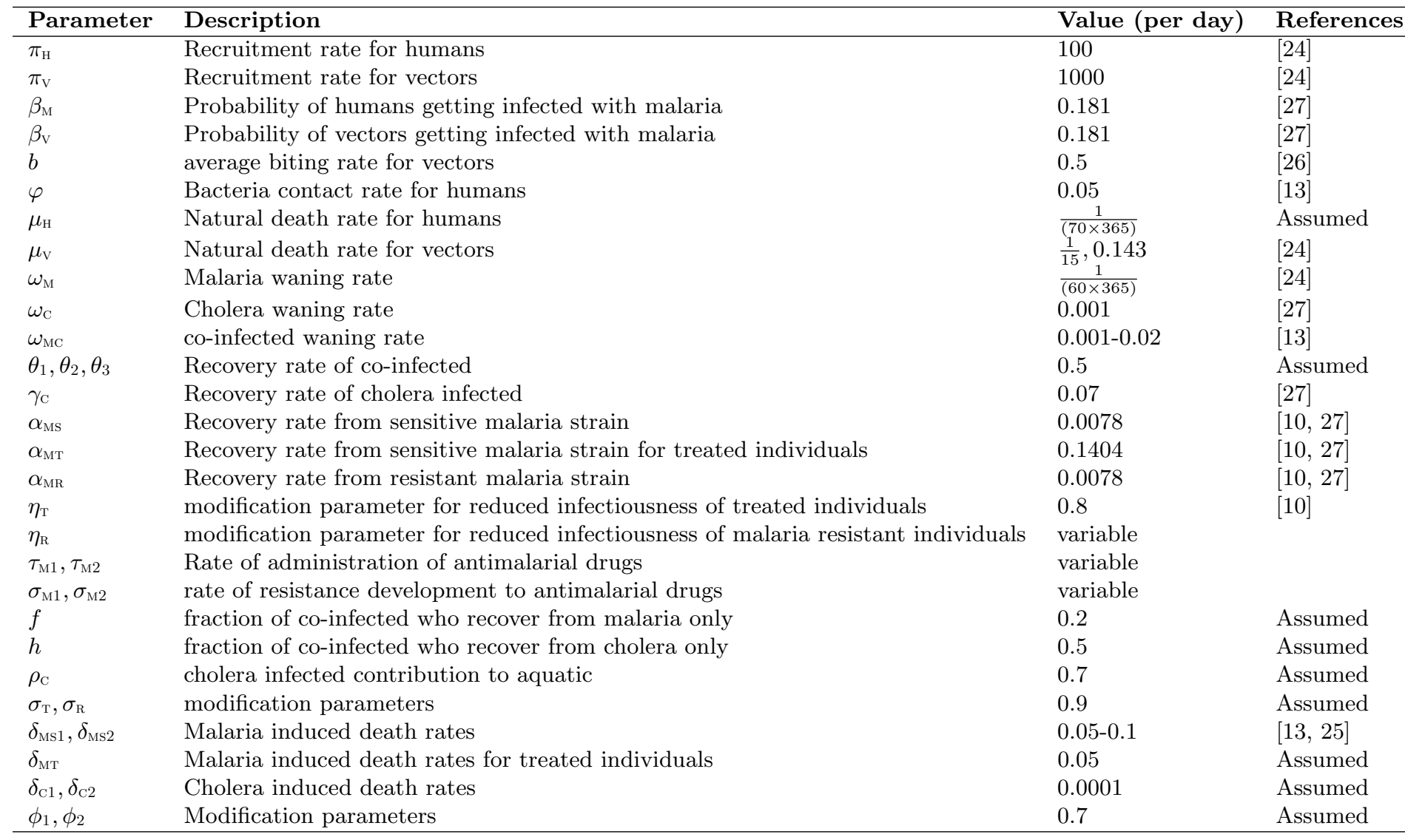




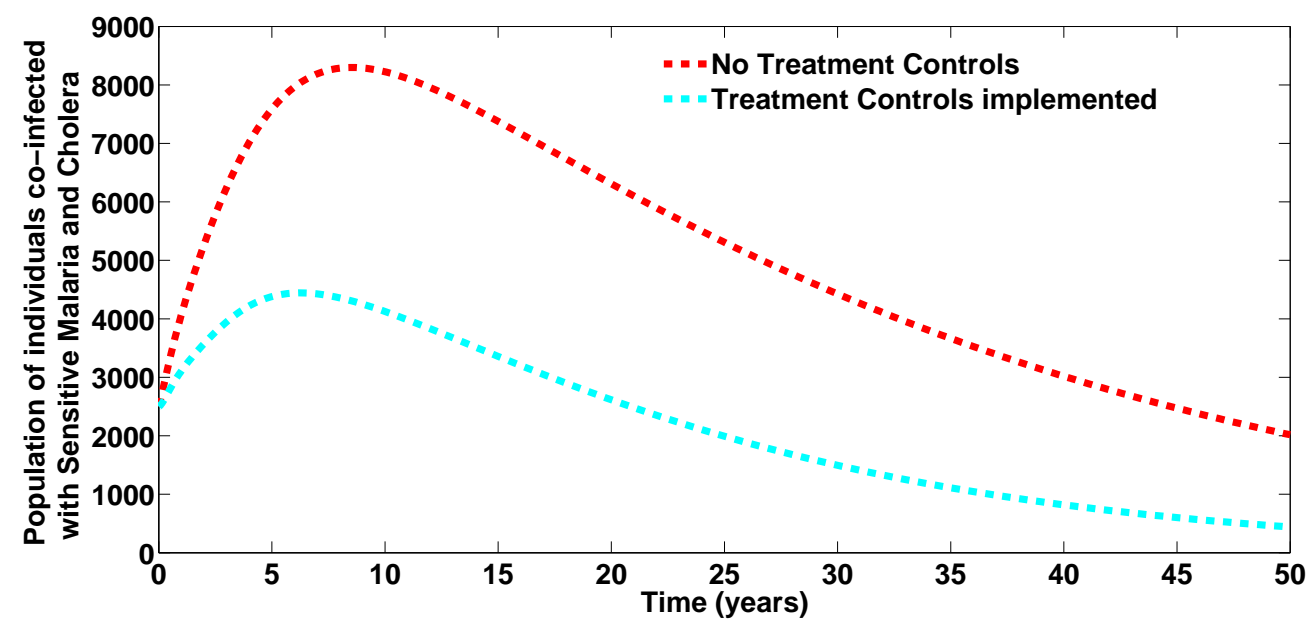

Figure 2: Simulations of the model (2) showing the total number of untreated of sensitive strain and co-infected with cholera, when there is no drug resistance. Here, $\sigma_{\mathrm{M} 1}=\sigma_{\mathrm{M} 2}=0$. All other parameters as in Table 2

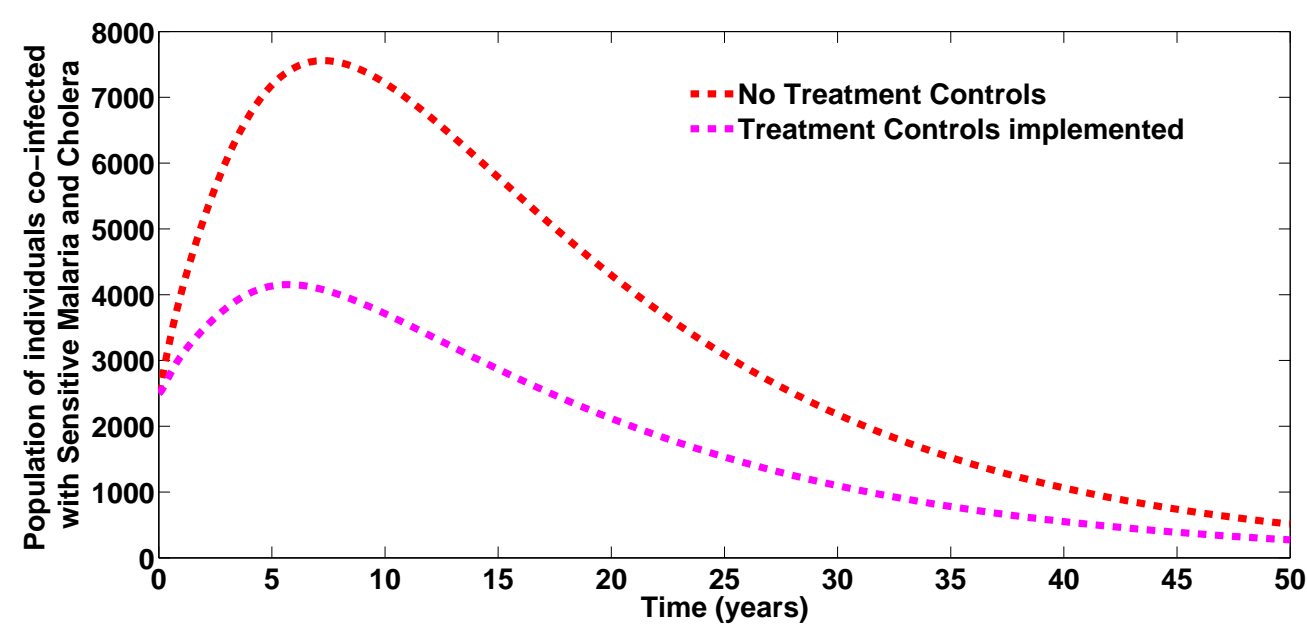

Figure 3: Simulations of the model (2) showing the total number of untreated of sensitive strain and co-infected with cholera, when there is drug resistance. Here, $\sigma_{\mathrm{M} 1}=\sigma_{\mathrm{M} 2}=0.4$. All other parameters as in Table 2

Figure 8 shows the simulation of the total number of infected individual at different initial conditions. It is observed that the resistant strain drives the sensitive strain to extinction when both reproduction numbers are greater than unity, with $\left(1<\mathcal{R}_{0 \mathrm{MS}}<\mathcal{R}_{0 \mathrm{MR}}\right)$. both strains co-exist with the sentitive strains dominating the resistaant strain when both reproduction number are greater than umity $(1<$ $\left.\mathcal{R}_{0 \mathrm{MR}}<\mathcal{R}_{0 \mathrm{MS}}\right)$. Likewise when both reproduction numbers are greater than unity with $\left(\mathcal{R}_{0 \mathrm{MS}}=3.54103 \approx\right.$ $\left.3.54688=\mathcal{R}_{0 \mathrm{MR}}\right)$, both strain co-exist with th sensitive strain dominating but not driving the resistant strain to extinction.

\section{Conclusion}

In this work, we have considered and analyzed a mathematical model for two strains of Malaria and Cholera with optimal control. The model assessed the impact of treatment controls in reducing the burden of the two diseases in a population, in the presence of malaria drug resistance. The model was shown to exhibit the dynamical property of backward bifurcation when the associated reproduction number is less than unity. The global asymptotic stability of the disease-free equilibrium of the model was proven not to exist. The necessary conditions for the existence of optimal control and the optimality system for the model is established using the Pontryagin's Maximum Principle. Numerical simulations 
medRxiv preprint doi: https://doi.org/10.1101/2020.08.18.20177329; this version posted September 10, 2020. The copyright holder for this preprint (which was not certified by peer review) is the author/funder, who has granted medRxiv a license to display the preprint in

It is made available under a CC-BY-NC-ND 4.0 International license .

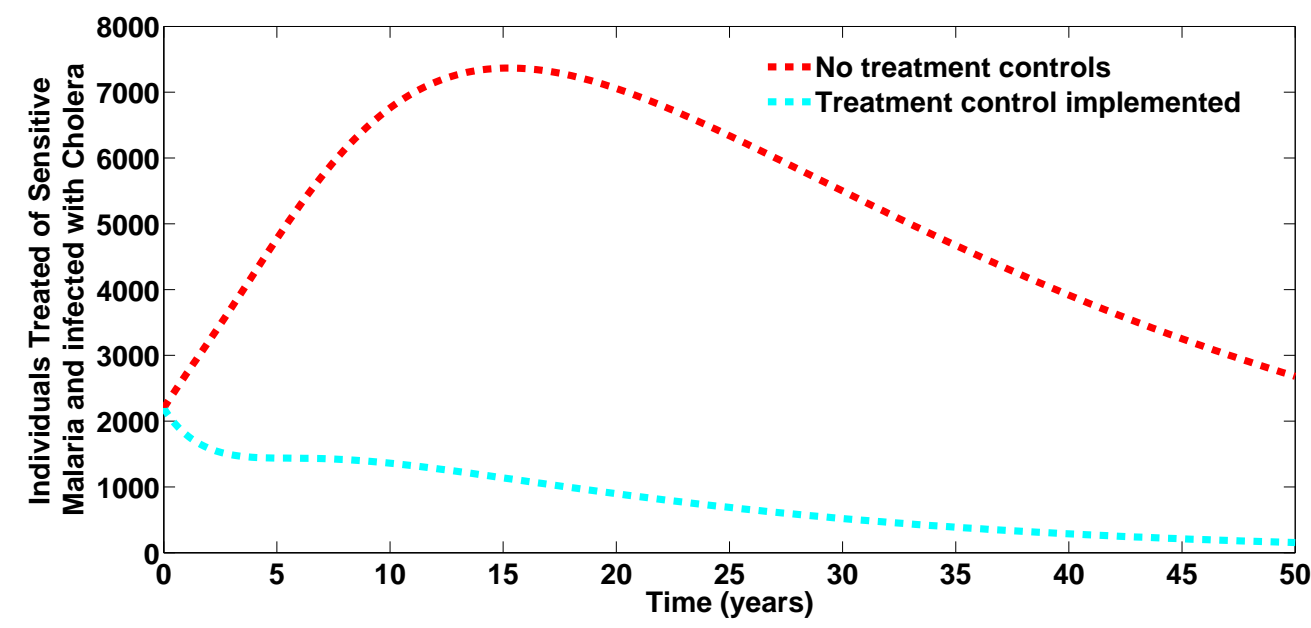

Figure 4: Simulations of the model (2) showing the total number of treated of sensitive strain and coinfected with cholera, when there is no drug resistance. Here, $\sigma_{\mathrm{M} 1}=\sigma_{\mathrm{M} 2}=0$. All other parameters as in Table 2

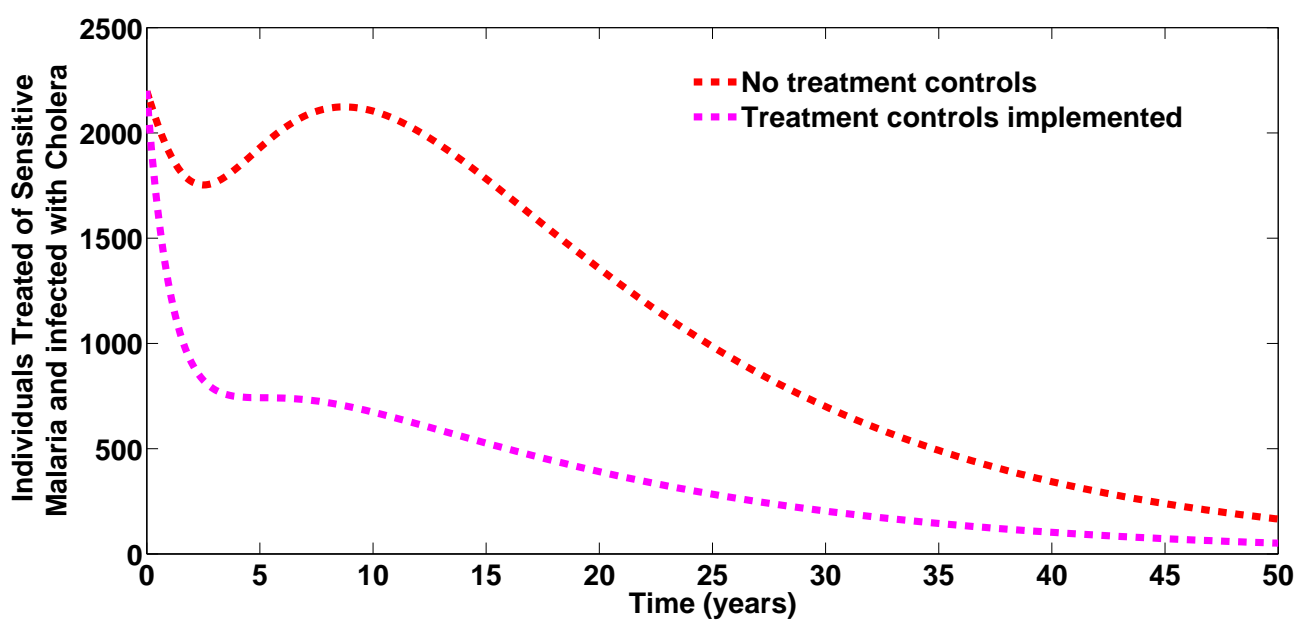

Figure 5: Simulations of the model (2) showing the total number of treated of sensitive strain and coinfected with cholera, when there is drug resistance. Here, $\sigma_{\mathrm{M} 1}=\sigma_{\mathrm{M} 2}=0.4$. All other parameters as in Table 2

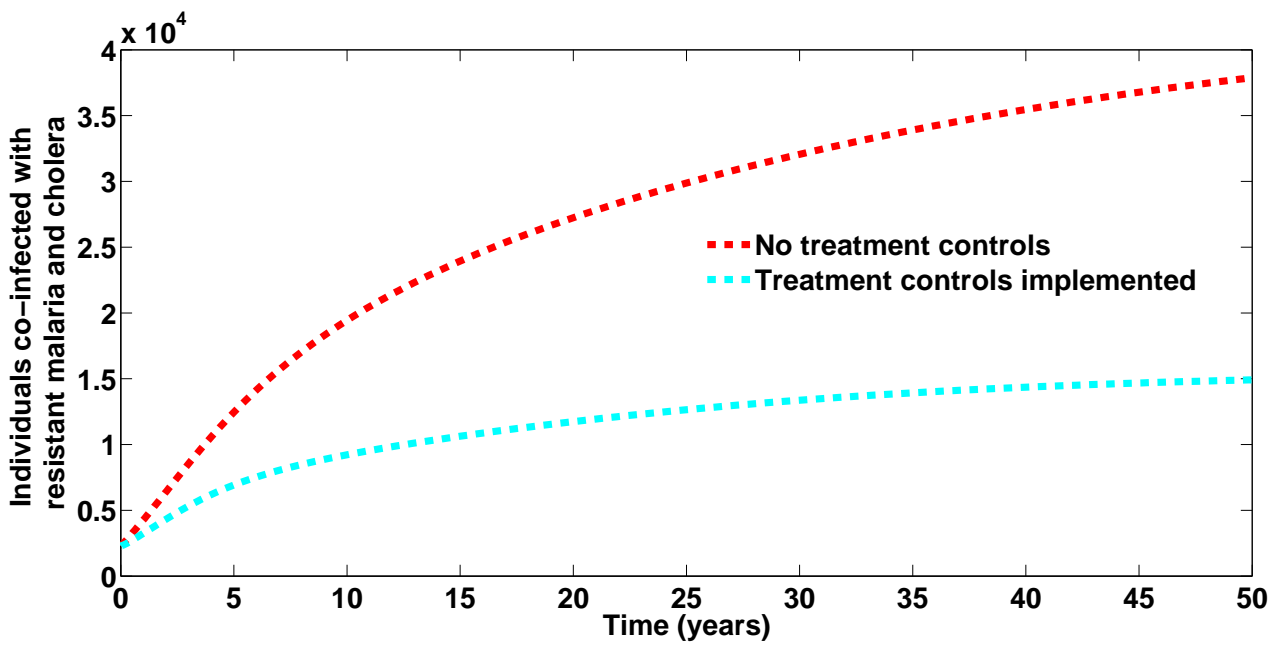

Figure 6: Simulations of the model (2) showing the total number of individuals co-infected with resistant malaria and cholera, when there is no drug resistance. Here, $\sigma_{\mathrm{M} 1}=\sigma_{\mathrm{M} 2}=0$. All other parameters as in Table 2 
medRxiv preprint doi: https://doi.org/10.1101/2020.08.18.20177329; this version posted September 10, 2020. The copyright holder for this preprint (which was not certified by peer review) is the author/funder, who has granted medRxiv a license to display the preprint in

It is made available under a CC-BY-NC-ND 4.0 International license .

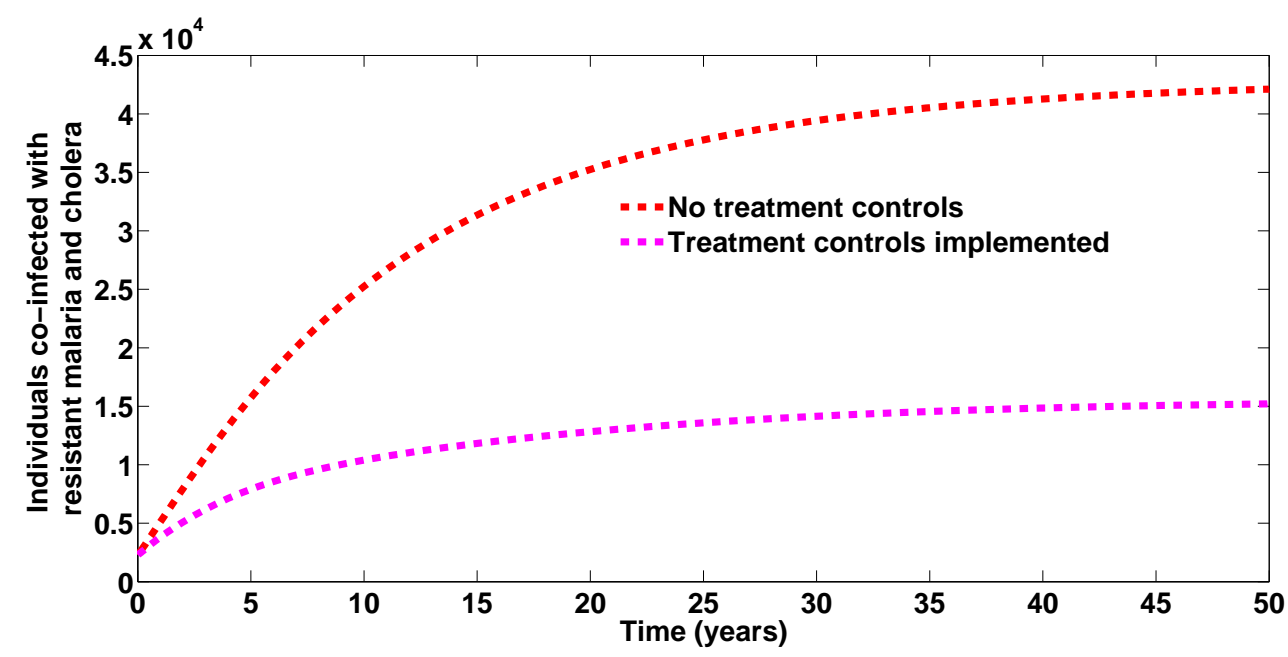

Figure 7: Simulations of the model (2) showing the total number of individuals co-infected with resistant malaria and cholera, when there is drug resistance. Here, $\sigma_{\mathrm{M} 1}=\sigma_{\mathrm{m} 2}=0.4$. All other parameters as in Table 2

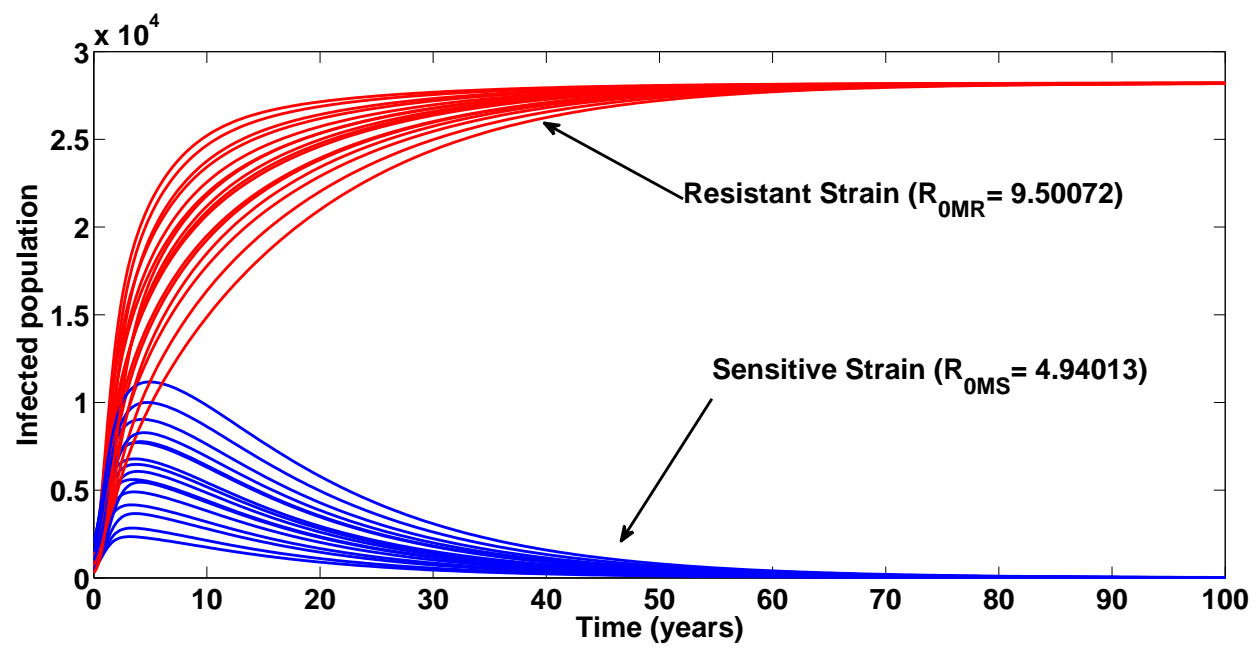

Figure 8: Simulations of the model (2) showing the total number of infected individuals at different initial conditions. Here, $\beta_{\mathrm{M}}=7.034, \beta_{7}=7.09, \eta_{\mathrm{R}}=1.2, \quad\left(\right.$ so that $\quad \mathcal{R}_{0 \mathrm{MS}}=4.94013<\mathcal{R}_{0 \mathrm{MR}}=9.50072$ ). All other parameters as in Table 2

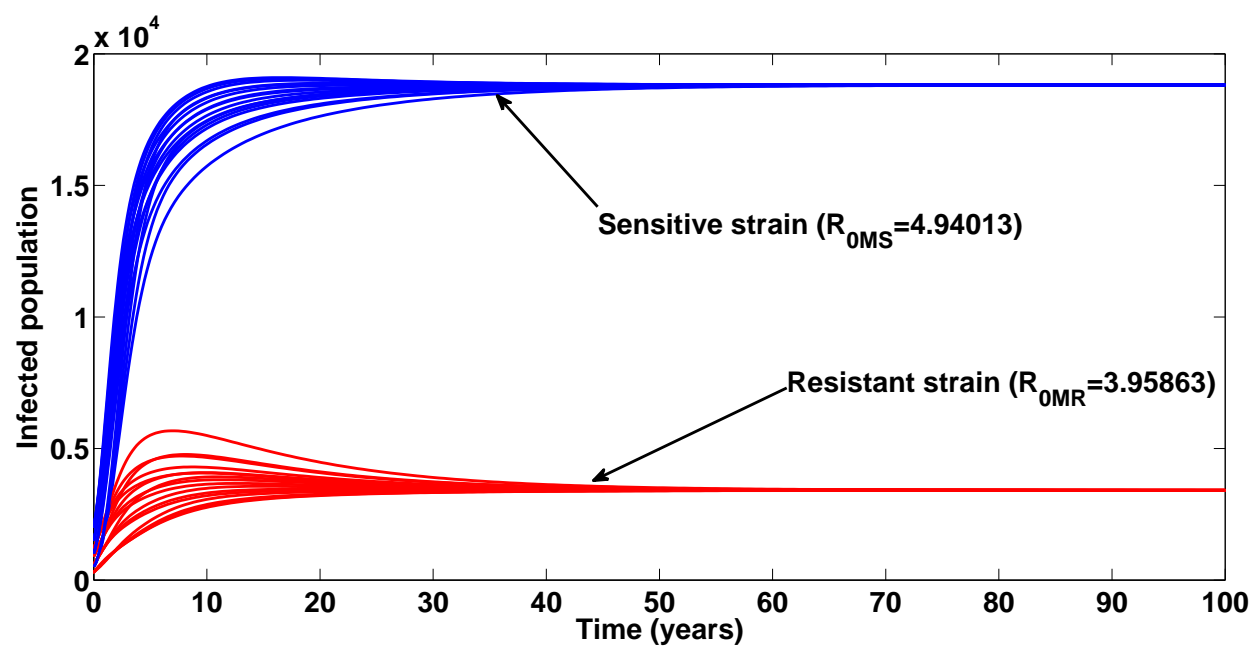

Figure 9: Simulations of the model (2) showing the total number of infected individuals at different initial conditions. Here, $\beta_{\mathrm{M}}=7.034, \beta_{7}=7.09, \eta_{\mathrm{R}}=0.5, \quad$ (so that $\quad \mathcal{R}_{0 \mathrm{MS}}=4.94013>\mathcal{R}_{0 \mathrm{MR}}=3.95863$ ). All other parameters as in Table 2 
medRxiv preprint doi: https://doi.org/10.1101/2020.08.18.20177329; this version posted September 10, 2020. The copyright holder for this preprint (which was not certified by peer review) is the author/funder, who has granted medRxiv a license to display the preprint in

It is made available under a CC-BY-NC-ND 4.0 International license .

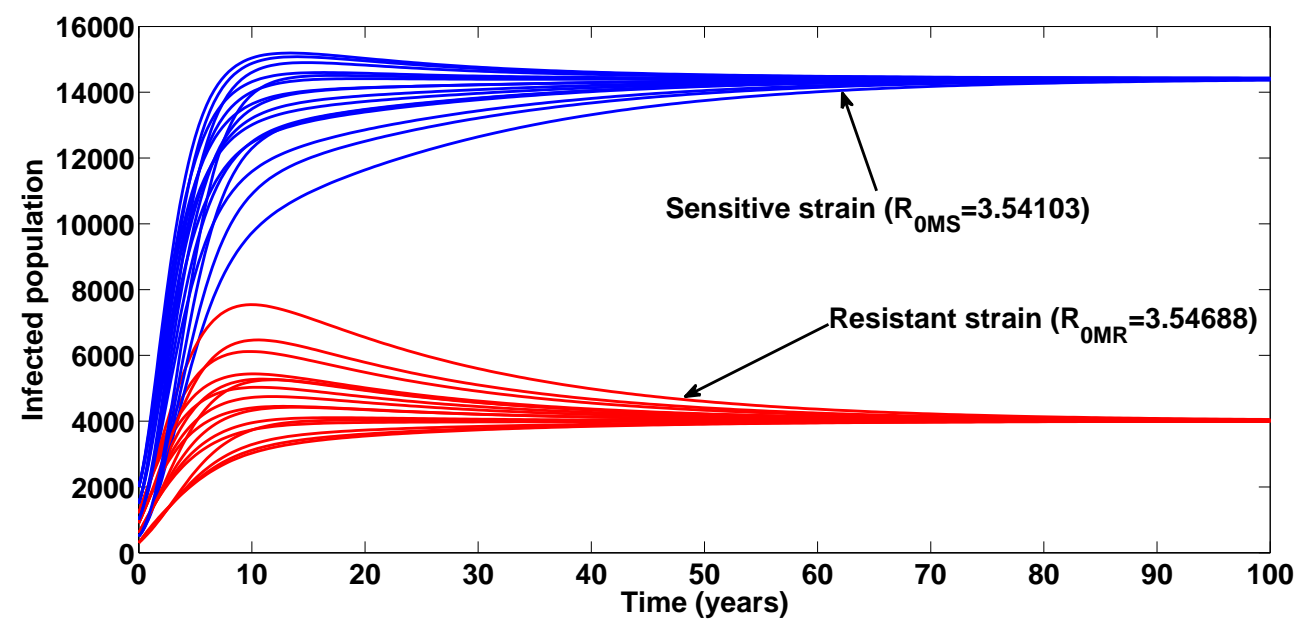

Figure 10: Simulations of the model (2) showing the total number of infected individuals at different initial conditions. Here, $\beta_{\mathrm{M}}=5.034, \beta_{\mathrm{v}}=5.09, \eta_{\mathrm{R}}=0.625, \quad\left(\right.$ so that $\left.\quad \mathcal{R}_{0 \mathrm{MS}}=3.954103 \approx \mathcal{R}_{0 \mathrm{MR}}=3.54688\right)$. All other parameters as in Table 2

of the optimal control model revealed that malaria drug resistance can greatly influence the co-infection cases averted, even in the presence of treatment controls for co-infected individuals 
medRxiv preprint doi: https://doi.org/10.1101/2020.08.18.20177329; this version posted September 10, 2020. The copyright holder for this preprint (which was not certified by peer review) is the author/funder, who has granted medRxiv a license to display the preprint in

It is made available under a CC-BY-NC-ND 4.0 International license .

\section{References}

[1] Birhanie M, Tessema B, Ferede G, Endris M, Enawgaw B (2014) Malaria Typhoid Fever, and Their Coinfection among Febrile Patients at a Rural Health Center in Northwest Ethiopia: A Cross-Sectional Study, Advances in Medicine Volume 2014, Article ID 531074, http://dx.doi.org/10.1155/2014/531074.

[2] The World malaria report 2019, https://www.who.int/malaria/publications/ world-malaria-report$2019 /$ en/

[3] Centres for Disease Control and Prevention (CDC) Cholera - Vibrio cholerae infection, https://www.cdc.gov/cholera/general/index.html. Accesed, 12th August, 2020.

[4] Okuonghae D, Omame A, Analysis of a mathematical model for COVID-19 population dynamics in Lagos, Nigeria, Chaos Solitons Fractals 2020;139:110032

[5] Omame A, Umana RA, Okuonghae D, Inyama SC, Mathematical analysis of a two-sex Human Papillomavirus (HPV) model, International Journal of Biomathematics, 11 (2018) (7)

[6] Omame A, Okuonghae D, Umana RA, Inyama SC, Analysis of a co-infection model for HPV-TB, Applied Mathematical Modelling, 77 (2020) 881-901.

[7] Omame A, Okuonghae D, Inyama SC, A mathematical study of a model for HPV with two high risk strains, in Mathematics Applied to Engineering, Modelling, and Social Issues Studies in Systems, Decision and Control (2020) 200, F. Smith, H. Dutta and J. N. Mordeson (eds.)

[8] Umana RA, Omame A, Inyama SC, Deterministic and Stochastic Models of the Dynamics of Drug Resistant Tuberculosis; FUTO Journals Series, (2016) 2 (2) 173-194.

[9] Uwakwe JI, Inyama SC, Omame A, Mathematical Model and Optimal Control of NewCastle Disease (ND). Applied and Computational Mathematics. (2020) 9 (3) 70-84. doi: 10.11648/j.acm.20200903.14

[10] Esteva L, Gumel AB, de Leon CV, Qualitative study of transmission dynamics of drug-resistant malaria Math. Comput. Model. 50 (2009) 611-630.

[11] Edward S, Nyerere N, A Mathematical Model for the Dynamics of Cholera with Control Measures, Appl, Comput. Math. 4(2);2015:53-63.

[12] Agusto FB, Adekunle AI, Optimal control of a two-strain tuberculosis-HIV/AIDS co-infection model, BioSystems, (2014) (119) 20-24.

[13] Okosun KO, Makinde OD, Optimal control analysis of malaria in the presence of non-linear incidence rate, Appl. Comput. Math. 12 (1) (2013) 20-32.

[14] Omame A, Sene N, Nometa I, Nwakanma CI, Nwafor EU, Iheonu NO, Okuonghae D, Analysis of COVID-19 and comorbidity co-infection Model with Optimal Control, medRxiv preprint doi: https://doi.org/10.1101/2020.08.04.20168013.

[15] Z. Mukandavire, A. B. Gumel, W. Garira and J.M Tchuenche, Mathematical Analysis of a Model for HIV-Malaria Co-infection, Math. Biosci. Eng. 6 (2) (2009) 333-362. 
medRxiv preprint doi: https://doi.org/10.1101/2020.08.18.20177329; this version posted September 10, 2020. The copyright holder for this preprint (which was not certified by peer review) is the author/funder, who has granted medRxiv a license to display the preprint in It is made available under a CC-BY-NC-ND 4.0 International license.

[16] S. Mushayabasa, J. M. Tchuenche, C. P. Bhunu and E. Ngarakana-Gwasira, Modeling gonorrhea and HIV co-interaction, BioSystems, 103 (1) (2011) 27-37.

[17] J. Naresh and A. Tripathi, Modelling and analysis of HIV-TB co-infection in a variable size population, Math. Model. Anal. 10 (3) (2005) 275-286.

[18] A. Nwankwo and D. Okuonghae, Mathematical analysis of the transmission dynamics of HIV Syphilis Co-infection in the presence of treatment for Syphilis, Bull. Math. Biol. 80 (3) (2018) 437-492.

[19] Lakshmikantham S, Leela S, Martynyuk AA, Stability Analysis of Nonlinear Systems, Marcel Dekker, Inc., New York, 1989.

[20] Hethcote HW, The mathematics of infectious diseases, SIAM Rev. 2000;42(4):599-653.

[21] van den Driessche P, Watmough J, Reproduction numbers and sub-threshold endemic equilibria for compartmental models of disease transmission, Math. Biosci. 180 (2002) 29-48.

[22] Castillo-Chavez C, Feng Z, Huang W, On the computation of $R_{0}$ and its role on global stability, in Mathematical Approaches for Emerging and Reemerging Infectious Diseases: An Introduction (Minneapolis, MN, 1999), 229-250, IMA Vol. Math. Appl., 125 Springer New York.

[23] Castillo-Chavez C, Song B, Dynamical models of tuberculosis and their applications, Math. Biosci. Eng. 2 (2004) 361-404.

[24] Blayneh KW, Cao Y, Kwon HD, Optimal control of vector-borne diseases: treatment and Prevention, Discrete Cont. Dyn. Syst. B11 (3) (2009) 587?611.

[25] Robert JS, Hove-Musekwa SD, Determining effective spraying periods to control malaria via indoor residual spraying in sub-saharan Africa, J. Appl. Math. Decis. Sci. (2008) ArticleID 745463, Hindawi Publishing Corporation.

[26] Aron JL, May RM, The Population Dynamics of Malaria, in: R.M. Anderson (Ed.), Population Dynamics of Infectious Diseases, Chapman and Hall, London, 1982, pp. 139-179.

[27] Ishikawa H, Ishii A, Nagai N, Ohmae H, Harada M, Suguri S, Leafasia J, A mathematical model for the transmission of Plasmodium vivax malaria, Parasitol. Internat. 52 (2003) 81-93.

[28] Pontryagin LS, Boltyanskii VG, Gamkrelidze RV, Mishchenko EF, The Mathematical Theory of Optimal Processes, Wiley, New York, 1962.

[29] Lenhart, S., Workman, J.T., Optimal Control Applied to Biological Models. Chapman \& Hall, Boca Raton, (2007). 